\title{
GENERAL MAZUR-ULAM TYPE THEOREMS AND SOME APPLICATIONS
}

\author{
LAJOS MOLNÁR \\ Dedicated to Professor Charles J.K. Batty on the occasion of his 60th birtday.
}

\begin{abstract}
Recently we have presented several structural results on certain isometries of spaces of positive definite matrices and on those of unitary groups. The aim of this paper is to put those previous results into a common perspective and extend them to the context of operator algebras, namely, to that of von Neumann factors.
\end{abstract}

\section{INTRODUCTION AND STATEMENT OF THE RESULTS}

The famous Mazur-Ulam theorem states that every surjective isometry (i.e., surjective distance preserving map) from a normed real linear space onto another one is automatically affine, in other words, it is necessarily an isomorphism with respect to the operation of convex combinations.

Recently we have extensively investigated how this fundamental theorem can be generalized to more general settings. In [12] we have obtained results in the context of groups (and some of their substructures) which state that under certain conditions surjective distance preserving transformations between such structures necessarily preserve locally the operation of the so-called inverted Jordan product. This means that also in that general setting the surjective isometries necessarily have a particular algebraic property. This property opens the way for employing algebraic ideas, techniques and computations to get more information about the isometries under considerations. In some of our latter papers we have successfully used that approach to describe explicitly the isometries of different non-linear structures of matrices and operators.

In [13] we have determined the surjective isometries of the unitary group over a Hilbert space equipped with the metric of the operator norm. In [21] we described the surjective isometries of the space of all positive definite operators on a Hilbert space relative to the so-called Thompson part metric. In [14] we have presented generalizations of the latter two results for the setting of $C^{*}$-algebras. It has turned out that the corresponding surjective isometries are closely related to Jordan *-isomorphisms between the underlying full algebras. In [25], [22] we

2010 Mathematics Subject Classification. Primary: 47B49. Secondary: 46L40.

Key words and phrases. Mazur-Ulam type theorems, generalized distance measures, positive definite cone, unitary group, Jordan triple map, inverted Jordan triple map, operator algebras.

The author was supported by the "Lendület" Program (LP2012-46/2012) of the Hungarian Academy of Sciences and by the Hungarian Scientific Research Fund (OTKA) Reg.No. K81166 NK81402. 
have proceeded further and described the structure of surjective isometries of the unitary group with respect to complete symmetric norms (see the definition later) both in the infinite and in the finite dimensional cases. Furthermore, in [22] we have also determined the isometries relative to the elements of a recently introduced collection of metrics [8] (having connections to quantum information science) on the group of unitary matrices. In [23] we have revealed the structure of surjective isometries of the space of positive definite matrices relative to certain metrics of differential geometric origin (they are common generalizations of the Thompson part metric and the natural Riemannian metric on positive definite matrices) as well as to a new metric obtained from the JensenShannon symmetrization of the important divergence called Stein's loss. In [26] we have made an important step toward further generality. Namely, we have described the structure of those surjective maps on the space of all positive definite matrices which leave invariant a given element of a large collection of certain so-called generalized distance measures. In that way we could present a common generalization of the mentioned results in [23] and also provide structural information on a large class of transformations preserving other particular important distance measures including Stein's loss itself. We also mention that by the help of appropriate modifications in our general results in [12] we have managed to determine the surjective isometries of Grassmann spaces of projections of a fixed rank on a Hilbert space relative to the gap metric [5].

In this paper we develop even further the ideas and approaches we have worked out and used in the papers [22], [23], [26], and extend our previous results concerning distance measure preserving maps on matrix algebras for the case of operator algebras, especially, von Neumann factors. We obtain results which show that if the positive definite cones or the unitary groups in those algebras equipped with a sort of very general distance measures are "isometric", then the underlying full algebras are Jordan *-isomorphic (either *-isomorphic or *-antiisomorphic).

We begin the presentation with the case of positive definite cones. As the starting point of the route leading to our corresponding result we exhibit a Mazur-Ulam type theorem for a certain very general structure called pointreflection geometry equipped with a generalized distance measure. In fact, we believe that with this result we have found in some sense the most general version of Mazur-Ulam theorem that one can obtain using the approach followed in [12] in the setting of groups. We point out that many of the arguments below use ideas that have already appeared in our previous papers [22], [23], [26]. In several cases only small changes need to be performed while in other cases we really have to work to find solutions for particular problems that emerge from the fact that instead of matrix algebras here we consider much more complicated objects, namely operator algebras. In order to make the material readable we present the results with complete proofs.

For our new general Mazur-Ulam type result we need the following concept that has been defined by Manara and Marchi in [19] (also see [16], [18]). 
Definition 1. Let $X$ be a set equipped with a binary operation $\diamond$ which satisfies the following conditions:

(a1) $a \diamond a=a$ holds for every $a \in X$;

(a2) $a \diamond(a \diamond b)=b$ holds for any $a, b \in X$;

(a3) the equation $x \diamond a=b$ has a unique solution $x \in X$ for any given $a, b \in X$.

In this case the pair $(X, \diamond)$ (or $X$ itself) is called a point-reflection geometry.

Observe that from the properties (a1)-(a2) above we easily obtain that the equation $a \diamond x=b$ also has unique solution $x \in X$ for any given $a, b \in X$.

As for our present purposes, the most important example of such a structure is given as follows. In the rest of the paper by a $C^{*}$-algebra we always mean a unital $C^{*}$-algebra with unit $I$. Let $\mathscr{A}$ be such an algebra. We denote by $\mathscr{A}_{s}$ the self-adjoint part of $\mathscr{A}$ and $\mathscr{A}_{+}$stands for the cone of all positive elements of $\mathscr{A}$ (self-adjoint elements with non-negative spectrum). The set of all invertible elements in $\mathscr{A}_{+}$is denoted by $\mathscr{A}_{+}^{-1}$. Sometimes $\mathscr{A}_{+}^{-1}$ is called positive definite cone and its elements are said positive definite. For any $A, B \in \mathscr{A}_{+}^{-1}$ define $A \diamond$ $B=A B^{-1} A$. In that way $\mathscr{A}_{+}^{-1}$ becomes a point-reflection geometry. Indeed, the conditions (a1), (a2) above are trivial to check. Concerning (a3) we recall that for any given $A, B \in \mathscr{A}_{+}^{-1}$, the so-called Ricatti equation $X A^{-1} X=B$ has a unique solution $X=A \# B$ which is just the geometric mean of $A$ and $B$ defined by

$$
A \# B=A^{1 / 2}\left(A^{-1 / 2} B A^{-1 / 2}\right)^{1 / 2} A^{1 / 2} .
$$

This assertion is usually termed as Anderson-Trapp theorem (for the original source see [1]).

In our general Mazur-Ulam type theorem that we are going to present we do not need to confine the considerations to true metrics, the theorem works also for so-called generalized distance measures.

Definition 2. Given an arbitrary set $X$, the function $d: X \times X \rightarrow[0, \infty[$ is called a generalized distance measure if it has the property that for an arbitrary pair $x, y \in X$ we have $d(x, y)=0$ if and only if $x=y$.

Hence, in the definition above we require only the definiteness property of a metric but neither the symmetry nor the triangle inequality is assumed. Our new general Mazur-Ulam type theorem reads as follows.

Theorem 3. Let $X, Y$ be sets equipped with binary operations $\diamond, \star$, respectively, with which they form point-reflection geometries. Let $d: X \times X \rightarrow[0, \infty[, \rho: Y \times$ $Y \rightarrow[0, \infty[$ be generalized distance measures. Pick $a, b \in X$, set

$$
L_{a, b}=\{x \in X: d(a, x)=d(x, b \diamond a)=d(a, b)\}
$$

and assume the following:

(b1) $d\left(b \diamond x, b \diamond x^{\prime}\right)=d\left(x^{\prime}, x\right)$ holds for all $x, x^{\prime} \in X$;

(b2) $\sup \left\{d(x, b): x \in L_{a, b}\right\}<\infty$;

(b3) there exists a constant $K>1$ such that $d(x, b \diamond x) \geq K d(x, b)$ holds for every $x \in L_{a, b}$ 
Let $\phi: X \rightarrow Y$ be a surjective map such that

$$
\rho\left(\phi(x), \phi\left(x^{\prime}\right)\right)=d\left(x, x^{\prime}\right), \quad x, x^{\prime} \in X
$$

and also assume that

(b4) for the element $c \in Y$ with $c \star \phi(a)=\phi(b \diamond a)$ we have $\rho\left(c \star y, c \star y^{\prime}\right)=$ $\rho\left(y^{\prime}, y\right)$ for all $y, y^{\prime} \in Y$.

Then we have

$$
\phi(b \diamond a)=\phi(b) \star \phi(a) .
$$

The maps $\phi$ appearing in the theorem may be called "generalized isometries". Moreover, observe that the above result trivially includes the original MazurUlam theorem. To see this, take normed real linear spaces $X, Y$ and a surjective isometry $\phi: X \rightarrow Y$. Define the operation $\diamond$ by $x \diamond x^{\prime}=2 x-x^{\prime}, x, x^{\prime} \in X$ and the operation $\star$ similarly. Let $d, \rho$ be the metrics corresponding to the norms on $X$ and $Y$. Selecting any pair $a, b$ of points in $X$, it is apparent that all conditions in the theorem are fulfilled and hence we have $\phi(2 b-a)=2 \phi(b)-\phi(a)$. It easily implies that $\phi$ respects the operation of the arithmetic mean from which it follows that $\phi$ respects all dyadic convex combinations and finally, by the continuity of $\phi$, we conclude that $\phi$ is affine.

The above result shows that maps which conserve the "distances " with respect to a pair of generalized distance measures respect a pair of algebraic operations in some sense. We emphasize that in the result above as well as in our other general Mazur-Ulam type results that appeared in [12], the isometries respect or, in other words, preserve algebraic operations only locally, for certain pairs $a, b$ of elements. In fact, in that generality nothing more can be expected. To see this, one may refer to groups equipped with the discrete metrics: any bijection between them is a surjective isometry but clearly not necessarily an isomorphism in any adequate sense. Nonetheless, even if only locally, surjective distance measure preserver transformations appearing in the above theorem do have a certain algebraic property. And in the cases what we consider in the present paper it turns out that they in fact have this property globally. Therefore, the problem of describing those distance measure preserver transformations can be transformed to the problem of describing certain algebraic isomorphisms. This is exactly the strategy we are going to follow below.

Let us proceed toward the first group of our results which concern transformations between the positive definite cones of $C^{*}$-algebras. Before presenting the results we need to make some preparations. By a symmetric norm on a $C^{*}$ algebra $\mathscr{A}$ we mean a norm $N$ for which $N(A X B) \leq\|A\| N(X)\|B\|$ holds for all $A, X, B \in \mathscr{A}$. Here and in what follows $\|$.$\| stands for the original norm on \mathscr{A}$ what we sometimes call operator norm. Whenever we speak about topological properties (convergence, continuity, etc.) without specifying the topology we always mean the norm topology of $\|$.$\| . We call a norm N$ on $\mathscr{A}$ unitarily invariant if $N(U A V)=N(A)$ holds for all $A \in \mathscr{A}$ and unitary $U, V \in \mathscr{A}$. Furthermore, a norm $N$ on $\mathscr{A}$ is said to be unitary similarity invariant if we have $N\left(U A U^{*}\right)=N(A)$ for 
all $A \in \mathscr{A}$ and unitary $U \in \mathscr{A}$. It is easy to see that any symmetric norm is unitarily invariant and it is trivial that every unitarily invariant norm is unitary similarity invariant. For several examples of complete symmetric norms on $B(H)$, the algebra of all bounded linear operators on a complex Hilbert space $H$, we refer to [7]. They include the so-called $(c, p)$-norms and, in particular, the Ky Fan $k$ norms. We mention that in that paper the authors use the expression "uniform norm" for symmetric norms. Apparently, the above examples provide examples of complete symmetric norms on any $C^{*}$-subalgebra of $B(H)$ and hence on von Neumann algebras, too.

Now, we recall that in [23] we have described the structure of isometries of the space $\mathbb{P}_{n}$ of all positive definite $n \times n$ complex matrices with respect to the metric defined by

$$
d_{N}(A, B)=N\left(\log A^{-1 / 2} B A^{-1 / 2}\right), \quad A, B \in \mathbb{P}_{n},
$$

where $N$ is a unitarily invariant norm on $\mathbb{M}_{n}$. (It is a well-known fact that on matrix algebras a norm is symmetric if and only if it is unitarily invariant, see Proposition IV.2.4 in [3]). The importance of that metric comes from its differential geometric background (it is a shortest path distance in a Finsler-type structure on $\mathbb{P}_{n}$ which generalizes its fundamental natural Riemann structure, for references see [23]). In the recent paper [26] we have presented a substantial extension of that result for the case where the logarithmic function in (1) is replaced by any continuous function $f:] 0, \infty[\rightarrow \mathbb{R}$ that satisfies

(c1) $f(y)=0$ holds if and only if $y=1$;

(c2) there exists a number $K>1$ such that

$$
\left.\left|f\left(y^{2}\right)\right| \geq K|f(y)|, \quad y \in\right] 0, \infty[.
$$

We must point out that with this replacement we usually get not a true metric, only a generalized distance measure. However in that way we cover the cases of many important concepts of matrix divergences whose preserver transformations could hence be explicitly described, for details see [26].

We now define that new class of generalized distance measures in the context of $C^{*}$-algebras. Let $\mathscr{A}$ be a $C^{*}$-algebra, $N$ a norm on $\left.\mathscr{A}, f:\right] 0, \infty[\rightarrow \mathbb{R}$ a given continuous function with property (c1). Define $d_{N, f}: \mathscr{A}_{+}^{-1} \times \mathscr{A}_{+}^{-1} \rightarrow[0, \infty[$ by

$$
d_{N, f}(A, B)=N\left(f\left(A^{1 / 2} B^{-1} A^{1 / 2}\right)\right), \quad A, B \in \mathscr{A}_{+}^{-1} .
$$

It is apparent that $d_{N, f}$ is a generalized distance measure.

We also need the following notions. If $\mathscr{A}$ is a $C^{*}$-algebra and $A, B \in \mathscr{A}$, then $A B A$ is called the Jordan triple product of $A$ and $B$ while $A B^{-1} A$ is said to be their inverted Jordan triple product. If $\mathscr{B}$ is another $C^{*}$-algebra and $\phi: \mathscr{A}_{+}^{-1} \rightarrow \mathscr{B}_{+}^{-1}$ is a map which satisfies

$$
\phi(A B A)=\phi(A) \phi(B) \phi(A), \quad A, B \in \mathscr{A}_{+}^{-1},
$$

then it is called a Jordan triple map. If $\phi: \mathscr{A}_{+}^{-1} \rightarrow \mathscr{B}_{+}^{-1}$ fulfills

$$
\phi\left(A B^{-1} A\right)=\phi(A) \phi(B)^{-1} \phi(A), \quad A, B \in \mathscr{A}_{+}^{-1},
$$


then $\phi$ is said to be an inverted Jordan triple map. A bijective Jordan triple map is called a Jordan triple isomorphism and a bijective inverted Jordan triple map is said to be an inverted Jordan triple isomorphism.

Applying Theorem 3 we shall prove the following result.

Theorem 4. Let $\mathscr{A}, \mathscr{B}$ be $C^{*}$-algebras with complete symmetric norms $N, M$, respectively. Assume $N$ satisfies $N(|A|)=N(A)$ for all $A \in \mathscr{A}$. Suppose $f, g:] 0, \infty[\rightarrow$ $\mathbb{R}$ are continuous functions both satisfying (c1) and $f$ also fulfilling (c2). Let $\phi: \mathscr{A}_{+}^{-1} \rightarrow \mathscr{B}_{+}^{-1}$ be a surjective map which respects the pair $d_{N, f}, d_{M, g}$ of generalized distance measures in the sense that

$$
d_{M, g}(\phi(A), \phi(B))=d_{N, f}(A, B), \quad A, B \in \mathscr{A}_{+}^{-1} .
$$

Then $\phi$ is a continuous inverted Jordan triple isomorphism, i.e., a continuous bijective map that satisfies

$$
\phi\left(A B^{-1} A\right)=\phi(A) \phi(B)^{-1} \phi(A), \quad A, B \in \mathscr{A}_{+}^{-1} .
$$

Having this result, the next natural step is to try to describe the structure of all continuous inverted Jordan triple isomorphisms between positive definite cones. This is exactly what we do. Observe that the inverted Jordan triple isomorphisms are closely related to Jordan triple isomorphisms which are much more common, they appear e.g. in pure ring theory, too (though there they are considered between full rings and usually assumed to be additive which is definitely not the case here). Indeed, if $\phi: \mathscr{A}_{+}^{-1} \rightarrow \mathscr{B}_{+}^{-1}$ is an inverted Jordan triple isomorphism, then elementary computation shows that the transformation $\psi()=.\phi(I)^{-1 / 2} \phi(.) \phi(I)^{-1 / 2}$ is a unital inverted Jordan triple isomorphism which can easily be seen to be a Jordan triple isomorphism. Recall a map is called unital if it sends the identity to the identity.

In the following theorem we describe the structure of continuous Jordan triple isomorphisms between the positive definite cones of von Neumann factors. A linear functional $l: \mathscr{A} \rightarrow \mathbb{C}$ on an algebra $\mathscr{A}$ is said to be tracial if it has the property $l(A B)=l(B A), A, B \in \mathscr{A}$.

Theorem 5. Assume $\mathscr{A}, \mathscr{B}$ are von Neumann algebras and $\mathscr{A}$ is a factor not of type $\mathrm{I}_{2}$. Let $\phi: \mathscr{A}_{+}^{-1} \rightarrow \mathscr{B}_{+}^{-1}$ be a continuous Jordan triple isomorphism. Then there is either an algebra ${ }^{*}$-isomorphism or an algebra ${ }^{*}$-antiisomorphism $\theta: \mathscr{A} \rightarrow \mathscr{B}$, a number $c \in\{-1,1\}$, and a continuous tracial linear functional $l: \mathscr{A} \rightarrow \mathbb{C}$ which is real valued on $\mathscr{A}_{s}$ and $l(I) \neq-c$ such that

$$
\phi(A)=e^{l(\log A)} \theta\left(A^{c}\right), \quad A \in \mathscr{A}_{+}^{-1} .
$$

Conversely, for any algebra ${ }^{*}$-isomorphism or algebra ${ }^{*}$-antiisomorphism $\theta: \mathscr{A} \rightarrow$ $\mathscr{B}$, number $c \in\{-1,1\}$, and continuous tracial linear functional $l: \mathscr{A} \rightarrow \mathbb{C}$ which is real valued on $\mathscr{A}_{s}$ and $l(I) \neq-c$, the above displayed formula (3) defines a continuous Jordan triple isomorphism between $\mathscr{A}_{+}^{-1}$ and $\mathscr{B}_{+}^{-1}$.

As for the tracial linear functional $l$ appearing above we mention the following. It is proven in [11] that in a properly infinite von Neumann algebra, every element is the sum of two commutators. This gives us that if $\mathscr{A}$ in the theorem 
is of one of the types $\mathrm{I}_{\infty}, \mathrm{II}_{\infty}$, III, then the functional $l$ above vanishes. However, if $\mathscr{A}$ is of type $\mathrm{I}_{n}$ or type $\mathrm{II}_{1}$, then due to the existence of a normalized trace, it really shows up.

After this we shall easily obtain our theorem on the structure of surjective maps between the positive definite cones of von Neumann factors which respect pairs of generalized distance measures. The statement reads as follows.

Theorem 6. Let $\mathscr{A}, \mathscr{B}$ be von Neumann algebras with complete symmetric norms $N, M$, respectively. Assume $f, g:] 0, \infty[\rightarrow \mathbb{R}$ are continuous functions both satisfying (c1) and $f$ also fulfilling (c2). Suppose that $\mathscr{A}$ is a factor not of type $\mathrm{I}_{2}$. Let $\phi: \mathscr{A}_{+}^{-1} \rightarrow \mathscr{B}_{+}^{-1}$ be a surjective map which respects the pair $d_{N, f}, d_{M, g}$ of generalized distance measures in the sense that

$$
d_{M, g}(\phi(A), \phi(B))=d_{N, f}(A, B), \quad A, B \in \mathscr{A}_{+}^{-1} .
$$

Then there is either an algebra ${ }^{*}$-isomorphism or an algebra *-antiisomorphism $\theta: \mathscr{A} \rightarrow \mathscr{B}$, a number $c \in\{-1,1\}$, an element $T \in \mathscr{B}_{+}^{-1}$ and a continuous tracial linear functional $l: \mathscr{A} \rightarrow \mathbb{C}$ which is real valued on $\mathscr{A}_{s}$ and $l(I) \neq-c$ such that

$$
\phi(A)=e^{l(\log A)} T \theta\left(A^{c}\right) T, \quad A \in \mathscr{A}_{+}^{-1} .
$$

In the case where $\mathscr{A}$ is an infinite factor, the linear functional l is in fact missing.

Let us emphasize the interesting consequence of the above theorem that if the positive definite cones of two von Neumann factors (not of type $\mathrm{I}_{2}$ ) are "isometric" in a very general sense (with respect a pair of generalized distance measures), than the underlying algebras are necessarily isomorphic or antiisomorphic as algebras.

The second part of our results concerns transformations between unitary groups. For any $C^{*}$-algebra $\mathscr{A}$, we denote its unitary group by $\mathscr{A}_{u}$. Similarly to the case of the positive definite cone, we are going to consider certain generalized distance measures on $\mathscr{A}_{u}$. Let $N$ be a norm on $\mathscr{A}$ and $f: \mathbb{T} \rightarrow \mathbb{C}$ a continuous function having zero exactly at 1 and define

$$
d_{N, f}(U, V)=N\left(f\left(U V^{-1}\right)\right), \quad U, V \in \mathscr{A}_{u} .
$$

Clearly, $d_{N, f}$ is a generalized distance measure on $\mathscr{A}_{u}$. In particular, when $f(z)=$ $z-1, z \in \mathbb{T}$, we obtain $d_{N, f}(U, V)=N(U-V), U, V \in \mathscr{A}_{u}$, i.e., the usual norm distance with respect to $N$.

In the paper [22] we have considered a recently defined collection of metrics on the group of $n \times n$ unitary matrices. To the definition we recall that for any unitary matrix $U$ we have a unique Hermitian matrix $H$ with spectrum in ] $-\pi, \pi]$ such that $U=\exp (i H)$. This $H$ is called the angular matrix of $U$. Now, for a given unitarily invariant norm $N$ on the algebra $\mathbb{M}_{n}$ of all $n \times n$ complex matrices the distance $d_{N}(U, V)$ between unitary matrices $U$ and $V$ is defined by $d_{N}(U, V)=N(H)$, where $H$ is the angular matrix of $U V^{-1}$. These metrics have been introduced and studied in [8] and the corresponding isometries have been determined in Theorem 4 in [22]. Observe that these metrics "almost" fit into the general framework we have presented in (6) above. Indeed, there $f$ should 
be the argument function on $\mathbb{T}$, but the problem is that this function is not continuous. Apparently, it did not cause any problem in [22] since there we have considered finite dimensional setting where the spectrum is finite and on such a set all functions can be viewed continuous.

As in the case of the positive definite cone, in order to obtain reasonable structural results on transformations between unitary groups that respect generalized distance measures, we need to require certain conditions on the generating continuous function $f: \mathbb{T} \rightarrow \mathbb{C}$. These are the following:

(d1) $f(y)=0$ holds if and only if $y=1$;

(d2) there exists a number $K>1$ such that

$$
\left|f\left(y^{2}\right)\right| \geq K|f(y)|
$$

holds for all $y \in \mathbb{T}$ from a neighborhood of 1 .

One may ask what is the reason for the locality in (d2). The trivial answer is that we want to cover the case of the function $f(z)=z-1, z \in \mathbb{T}$ which corresponds to the usual norm distance.

Our result parallel to Theorem 4 which states that the "generalized isometries" between unitary groups of von Neumann algebras are continuous inverted Jordan triple isomorphisms is formulated below. To this we note that having a look at the concepts relating to maps on the positive definite cone which are given before Theorem 4 , the notions of Jordan triple maps, inverted Jordan triple maps, Jordan triple isomorphisms, inverted Jordan triple isomorphisms between unitary groups should be self-explanatory.

Theorem 7. Let $\mathscr{A}, \mathscr{B}$ be von Neumann algebras with complete symmetric norms $N, M$, respectively. Assume $f, g: \mathbb{T} \rightarrow \mathbb{R}$ are continuous functions having the property (d1) and $f$ also satisfies (d2). Let $\phi: \mathscr{A}_{u} \rightarrow \mathscr{B}_{u}$ be a surjective map that respects the pair $d_{N, f}, d_{M, g}$ of generalized distance measures in the sense that

$$
d_{M, g}(\phi(U), \phi(V))=d_{N, f}(U, V), \quad U, V \in \mathscr{A}_{u} .
$$

Then $\phi$ is a continuous inverted Jordan triple isomorphism, i.e., a continuous bijective map which satisfies

$$
\phi\left(U V^{-1} U\right)=\phi(U) \phi(V)^{-1} \phi(U), \quad U, V \in \mathscr{A}_{u} .
$$

Just as in the first part of this section, one can easily see that inverted Jordan triple maps between unitary groups are closely related to Jordan triple maps. In particular, if $\phi: \mathscr{A}_{u} \rightarrow \mathscr{B}_{u}$ is an inverted Jordan triple isomorphism, then it is apparent that the map $\psi()=.\phi(I)^{-1} \phi($.) is again an inverted Jordan triple isomorphism which is unital and hence it is a Jordan triple isomorphism. The following theorem describes the structure of continuous such isomorphisms in the case of von Neumann factors. In the course of its proof we employ an argument involving one-parameter unitary groups.

Theorem 8. Assume $\mathscr{A}, \mathscr{B}$ are von Neumann algebras. Let $\phi: \mathscr{A}_{u} \rightarrow \mathscr{B}_{u}$ be a continuous Jordan triple isomorphism, i.e., a continuous bijective map which satisfies

$$
\phi(U V U)=\phi(U) \phi(V) \phi(U), \quad U, V \in \mathscr{A}_{u} .
$$


Then there is either an algebra *-isomorphism or an algebra *-antiisomorphism $\theta: \mathscr{A} \rightarrow \mathscr{B}$ and scalars $c, d \in\{-1,1\}$ such that

$$
\phi(A)=d \theta\left(A^{c}\right), \quad A \in \mathscr{A}_{u} .
$$

Combining the above two results we can readily obtain our theorem on generalized distance measure preserving maps between unitary groups of von Neumann factors which reads as follows.

Theorem 9. Let $\mathscr{A}, \mathscr{B}$ be von Neumann algebras with complete symmetric norms $N, M$, respectively. Assume $f, g: \mathbb{T} \rightarrow \mathbb{R}$ are continuous functions having the property (d1) and $f$ also satisfies (d2). Let $\phi: \mathscr{A}_{u} \rightarrow \mathscr{B}_{u}$ be a surjective map that respects the pair $d_{N, f}, d_{M, g}$ of generalized distance measures in the sense that

$$
d_{M, g}(\phi(U), \phi(V))=d_{N, f}(U, V), \quad U, V \in \mathscr{A}_{u} .
$$

Then there is either an algebra *isomorphism or an algebra *antiisomorphism $\theta: \mathscr{A} \rightarrow \mathscr{B}$, a unitary element $W \in \mathscr{B}_{u}$ and a number $c \in\{-1,1\}$ such that

$$
\phi(U)=W \theta\left(U^{c}\right), \quad A \in \mathscr{A}_{u} .
$$

Just as in the case of the positive definite cone, we point out the interesting consequence of the above theorem that if the unitary groups of two von Neumann factors are "isometric" in a very general sense (with respect to some pair of generalized distance measures), then the underlying algebras are necessarily isomorphic or antiisomorphic as algebras.

\section{Proofs}

This section is devoted to the proofs of our results.

We begin with the proof of our new general Mazur-Ulam type theorem, Theorem 3. In fact, the argument we use here follows closely the ideas given in the proofs of Proposition 9, Lemma 10 and Proposition 11 in [26] which statements have been formulated in the context of so-called twisted subgroups of groups. The main novelty here is that we have found in a sense the most general structure (point-reflection geometry) for which that argument can be employed.

The first step toward the proof of Theorem 3 is the following lemma that appeared in [26] as Lemma 8. The proof is so short that for the sake of completeness we repeat it here.

Lemma 10. Let $X$ be a set and $d: X \times X \rightarrow[0, \infty[$ an arbitrary function. Assume $\varphi: X \rightarrow X$ is a bijective map satisfying

$$
d\left(\varphi(x), \varphi\left(x^{\prime}\right)\right)=d\left(x^{\prime}, x\right), \quad x, x^{\prime} \in X .
$$

Assume further that we have $b \in X$ for which $\sup \{d(x, b) \mid x \in X\}<\infty$, and there is a constant $K>1$ such that

$$
d(x, \varphi(x)) \geq K d(x, b), \quad x \in X .
$$

Then for every bijective map $f: X \rightarrow X$ satisfying

$$
d\left(f(x), f\left(x^{\prime}\right)\right)=d\left(x^{\prime}, x\right), \quad x, x^{\prime} \in X
$$

we have $d(f(b), b)=0$. 
Proof. For temporary use we call a map $f: X \rightarrow X d$-reversing if it satisfies (10). Let

$$
\lambda=\sup \{d(f(b), b) \mid f: X \rightarrow X \text { is a bijective } d \text {-reversing map }\} .
$$

Then $0 \leq \lambda<\infty$. For an arbitrary bijective $d$-reversing map $f: X \rightarrow X$, consider $\tilde{f}=f^{-1} \circ \varphi \circ f$. Then $\tilde{f}$ is also a bijective $d$-reversing transformation and

$$
\lambda \geq d(\tilde{f}(b), b)=d(f(b), \varphi(f(b))) \geq K d(f(b), b) .
$$

By the definition of $\lambda$ we get $\lambda \geq K \lambda$ which implies that $\lambda=0$ and this completes the proof.

The next proposition in the case where $X=Y, d=\rho$ appeared as Proposition 9 in [26].

Proposition 11. Let $X$ be a set and $d: X \times X \rightarrow[0, \infty[$ any function. Let $a, b \in$ $X$ and assume that $\varphi: X \rightarrow X$ is a bijective map which satisfies (9). Moreover, assume that $\varphi(b)=b$ and the composition map $\varphi \circ \varphi$ equals the identity on $X$. Set

$$
L=\{x \in X \mid d(a, x)=d(x, \varphi(a))=d(a, b)\} .
$$

Suppose that $\sup \{d(x, b) \mid x \in L\}<\infty$ and there exists a constant $K>1$ such that

$$
d(x, \varphi(x)) \geq K d(x, b), \quad x \in L .
$$

Let $Y$ be another set and $\rho: Y \times Y \rightarrow[0, \infty[$ any function. Assume $\psi: Y \rightarrow Y$ is a bijective map such that

$$
\rho\left(\psi(y), \psi\left(y^{\prime}\right)\right)=d\left(y^{\prime}, y\right), \quad y, y^{\prime} \in Y .
$$

If $T: X \rightarrow Y$ is a bijective map satisfying

$$
\rho\left(T(x), T\left(x^{\prime}\right)\right)=d\left(x, x^{\prime}\right), \quad x, x^{\prime} \in X,
$$

and

$$
\psi(T(a))=T(\varphi(a)), \quad \psi(T(\varphi(a)))=T(a)
$$

then we have

$$
\rho(\psi(T(b)), T(b))=0 .
$$

Proof. Since $\varphi(b)=b$ and $\varphi$ satisfies (9), we have

$$
d(a, b)=d(\varphi(b), \varphi(a))=d(b, \varphi(a))
$$

which implies that $b \in L$. Let

$$
L^{\prime}=\{y \in Y \mid \rho(T(a), y)=\rho(y, T(\varphi(a)))=d(a, b)\} .
$$

By the bijectivity and the property (11) of $T$ one can easily check that $T(L)=L^{\prime}$. Furthermore, using corresponding properties of the maps $\varphi, \psi$ as well as the intertwining properties (12), we obtain that $\varphi(L)=L$ and $\psi\left(L^{\prime}\right)=L^{\prime}$. Consider now the transformation $\tilde{T}=T^{-1} \circ \psi \circ T$. Plainly, the restriction of this map onto $L$ is a self-bijection of $L$ and it satisfies

$$
\rho\left(\tilde{T}(x), \tilde{T}\left(x^{\prime}\right)\right)=d\left(x^{\prime}, x\right), \quad x, x^{\prime} \in L .
$$


Since $\sup \{d(x, b) \mid x \in L\}<\infty$, we can apply Lemma 10 and deduce that

$$
0=d(\tilde{T}(b), b)=d(\psi(T(b)), T(b)) .
$$

After this preparation we can present the proof of our general Mazur-Ulam type result.

Proof of Theorem 3. First observe that by the definiteness of generalized distance measures the surjective "generalized isometry" $\phi$ is also injective. Let $\varphi(x)=b \diamond x$ for every $x \in X$ and define $\psi: Y \rightarrow Y$ by $\psi(y)=c \star y, y \in Y$. By the properties of point-reflection geometries and the assumptions in the theorem, $\varphi$ is a bijective map on $X$ and $\psi$ is a bijective map on $Y$, moreover all conditions appearing in Proposition 11 are easily seen to be satisfied with $\phi$ in the place of $T$. In fact, we obviously have $\psi(\phi(a))=\phi(\varphi(a))$ which, by taking into account that $\psi$ is an involution, implies that $\psi(\phi(\varphi(a)))=\phi(a)$. Applying Proposition 11 we get that $\rho(\psi(\phi(b)), \phi(b))=0$ which implies $\phi(b)=\psi(\phi(b))=c \star \phi(b)$. By the properties (a1), (a3) of point-reflection geometries we infer that $c=\phi(b)$ implying

$$
\phi(b \diamond a)=\phi(b) \star \phi(a) .
$$

In the next proposition on which the proof of Theorem 4 relies we shall need the following lemma about the monotonicity of symmetric norms. We believe its content is well-known but we could not find it in the literature. Therefore, we present it with a short proof.

Lemma 12. Let $N$ be a symmetric norm on a $C^{*}$-algebra $\mathscr{A}$ and assume $A, B \in \mathscr{A}_{+}$ are such that $A \leq B$. Then we have $N(A) \leq N(B)$.

Proof. Suppose first that $B$ is invertible. Let $D$ be the geometric mean of $B^{-1}$ and $A$, i.e.,

$$
D=B^{-1} \# A=B^{-1 / 2}\left(B^{1 / 2} A B^{1 / 2}\right)^{1 / 2} B^{-1 / 2} .
$$

Clearly, we have $A=D B D$ and observe that $D \leq I$ (this can be proven directly using the operator monotonicity of the square-root function, or referring to the monotonicity property of general operator means of Kubo-Ando sense). It follows that

$$
N(A)=N(D B D) \leq\|D\| N(B)\|D\| \leq N(B) .
$$

For non-invertible $B$, consider $B+\epsilon I$ for positive numbers $\epsilon$ tending to 0 .

Before presenting the next result we point out the easy fact that for any function $f:] 0, \infty[\rightarrow \mathbb{R}$ with the properties (c1) and (c2) we necessarily have

$$
\lim _{y \rightarrow 0}|f(y)|=\lim _{y \rightarrow \infty}|f(y)|=\infty .
$$

We also remark the following. If $f$ is a continuous scalar valued function on the set of positive real numbers, then for any $A \in \mathscr{A}_{+}^{-1}$ and unitary $U \in \mathscr{A}$ we have $f\left(U A U^{*}\right)=U f(A) U^{*}$. This is obviously true if $f$ is a polynomial and then one can refer to the fact that any continuous function on a compact interval 
can be uniformly approximated by polynomials to obtain the general statement. For any unitary similarity invariant norm $N$ on $\mathscr{A}$, it follows that $N\left(f\left(U A U^{*}\right)\right)=$ $N(f(A))$ holds for all $A \in \mathscr{A}$ and unitary $U \in \mathscr{A}$.

Finally, we admit that if $N$ is a complete symmetric norm on the $C^{*}$-algebra $\mathscr{A}$, then it is necessarily equivalent to the operator norm. Indeed, we have $N(A) \leq N(I)\|A\|, A \in \mathscr{A}$ and then the equivalence follows from the completeness of $N$ and $\|$.$\| .$

We are now in a position to prove the following proposition.

Proposition 13. Let $\mathscr{A}$ be a $C^{*}$-algebra with complete symmetric norm $N$ such that $N(|A|)=N(A)$ holds for all $A \in \mathscr{A}$. Assume $f:] 0, \infty[\rightarrow \mathbb{R}$ is a continuous function satisfying (c1), (c2). Consider the standard point-reflection geometry operation on $\mathscr{A}_{+}^{-1}$, i.e., let $A \diamond B=A B^{-1} A, A, B \in \mathscr{A}_{+}^{-1}$. Define $d_{N, f}$ as in (2). Then for the structure $\mathscr{A}_{+}^{-1}$ equipped with this operation $\diamond$ and generalized distance measure $d_{N, f}$ the assumptions (b1)-(b3) in Theorem 3 are satisfied for every pair $A, B \in \mathscr{A}_{+}^{-1}$. Moreover, for a sequence $\left(X_{n}\right)$ in $\mathscr{A}_{+}^{-1}$ and element $X \in \mathscr{A}_{+}^{-1}$ we have $X_{n} \rightarrow X$ in the operator norm topology if and only if $d_{N, f}\left(X, X_{n}\right) \rightarrow 0$.

Proof. Essentially, we follow the argument given in the proof of Theorem 1 in [26].

Pick $A, B \in \mathscr{A}_{+}^{-1}$ and consider the polar decomposition $B^{-1 / 2} A^{1 / 2}=U\left|B^{-1 / 2} A^{1 / 2}\right|$. Observe that by the invertibility of $A, B$ such a unitary $U \in \mathscr{A}$ does exist. We see that

$$
B^{-1 / 2} A B^{-1 / 2}=U\left|B^{-1 / 2} A^{1 / 2}\right|^{2} U^{*}=U A^{1 / 2} B^{-1} A^{1 / 2} U^{*} .
$$

Now, take an arbitrary invertible element $T \in \mathscr{A}$. Set

$$
X=A^{-1 / 2} B T^{*}\left(T A T^{*}\right)^{-1 / 2} .
$$

We deduce

$$
X X^{*}=A^{-1 / 2} B A^{-1} B A^{-1 / 2}=\left(A^{-1 / 2} B A^{-1 / 2}\right)^{2} .
$$

Consider the polar decomposition $X=V|X|, V \in \mathscr{A}$ being unitary. We compute

$$
\begin{gathered}
\left(T A T^{*}\right)^{-1 / 2} T B T^{*}\left(T A T^{*}\right)^{-1 / 2} \\
=\left(\left(T A T^{*}\right)^{-1 / 2} T B T^{*}\left(T A T^{*}\right)^{-1} T B T^{*}\left(T A T^{*}\right)^{-1 / 2}\right)^{1 / 2} \\
=\left(\left(T A T^{*}\right)^{-1 / 2} T B A^{-1} B T^{*}\left(T A T^{*}\right)^{-1 / 2}\right)^{1 / 2} \\
=\left(X^{*} X\right)^{1 / 2}=|X|=V^{*}\left|X^{*}\right| V=V^{*}\left(A^{-1 / 2} B A^{-1 / 2}\right) V .
\end{gathered}
$$

Recall that the generalized distance measure $d_{N, f}$ is defined by

$$
d_{N, f}(A, B)=N\left(f\left(A^{1 / 2} B^{-1} A^{1 / 2}\right)\right), \quad A, B \in \mathscr{A}_{+}^{-1} .
$$


Pick $B, X, Y \in \mathscr{A}_{+}^{-1}$. It follows from the content of (14) that for some unitary $V \in \mathscr{A}$ we have

$$
\begin{gathered}
(B \diamond X)^{1 / 2}(B \diamond Y)^{-1}(B \diamond X)^{1 / 2} \\
=\left(B X^{-1} B\right)^{1 / 2}\left(B Y^{-1} B\right)^{-1}\left(B X^{-1} B\right)^{1 / 2} \\
=\left(B^{-1} X B^{-1}\right)^{-1 / 2}\left(B^{-1} Y B^{-1}\right)\left(B^{-1} X B^{-1}\right)^{-1 / 2} \\
=V\left(X^{-1 / 2} Y X^{-1 / 2}\right) V^{*} .
\end{gathered}
$$

By (13), $X^{-1 / 2} Y X^{-1 / 2}$ is unitarily similar to $Y^{1 / 2} X^{-1} Y^{1 / 2}$ and hence we obtain the unitary similarity of $(B \diamond X)^{1 / 2}(B \diamond Y)^{-1}(B \diamond X)^{1 / 2}$ to $Y^{1 / 2} X^{-1} Y^{1 / 2}$. As we have mentioned above $N\left(f\left(U A U^{*}\right)\right)=N(f(A))$ holds for all $A \in \mathscr{A}_{+}^{-1}$ and unitary $U \epsilon$ $\mathscr{A}$. These observations imply that

$$
d_{N, f}(B \diamond X, B \diamond Y)=d_{N, f}(Y, X)
$$

holds for any $B, X, Y \in \mathscr{A}_{+}^{-1}$. Hence condition (b1) in Theorem 3 is fulfilled.

As for condition (b2), let us consider the set $\mathscr{H}$ of those elements $X \in \mathscr{A}_{+}^{-1}$ for which we have

$$
\begin{gathered}
d_{N, f}(A, X)=N\left(f\left(A^{1 / 2} X^{-1} A^{1 / 2}\right)\right) \\
=N\left(f\left(A^{1 / 2} B^{-1} A^{1 / 2}\right)\right)=d_{N, f}(A, B) .
\end{gathered}
$$

(With the notation of Theorem 3 we clearly have $L_{A, B} \subset \mathcal{H}$.) We show that the corresponding set of numbers

$$
\begin{gathered}
d_{N, f}(X, B)=N\left(f\left(X^{1 / 2} B^{-1} X^{1 / 2}\right)\right) \\
=N\left(f\left(B^{-1 / 2} X B^{-1 / 2}\right)\right)
\end{gathered}
$$

is bounded. Indeed, since $N\left(f\left(A^{1 / 2} X^{-1} A^{1 / 2}\right)\right)$ is constant on $\mathscr{H}$ and $N$ is equivalent to the operator norm $\|$.$\| , the set$

$$
\left\{\left\|f\left(A^{1 / 2} X^{-1} A^{1 / 2}\right)\right\|: X \in \mathscr{H}\right\}
$$

is bounded. We have already mentioned that $|f(y)| \rightarrow \infty$ as $y \rightarrow 0$ or $y \rightarrow$ $\infty$. It follows easily that there are positive numbers $m, M$ such that $m I \leq$ $A^{1 / 2} X^{-1} A^{1 / 2} \leq M I$ holds for all $X \in \mathscr{H}$. Clearly, we then have another pair $m^{\prime}, M^{\prime}$ of positive numbers such that $m^{\prime} I \leq X \leq M^{\prime} I$ and finally another one $m^{\prime \prime}, M^{\prime \prime}$ such that $m^{\prime \prime} I \leq B^{-1 / 2} X B^{-1 / 2} \leq M^{\prime \prime} I$ holds for all $X \in \mathscr{H}$. Equivalently, $m^{\prime \prime} I \leq X^{1 / 2} B^{-1} X^{1 / 2} \leq M^{\prime \prime} I$ holds for each $X \in \mathcal{H}$. By continuity, $f$ is bounded on the interval $\left[m^{\prime \prime}, M^{\prime \prime}\right]$ and this implies that the set

$$
\left\{N\left(f\left(X^{1 / 2} B^{-1} X^{1 / 2}\right)\right): X \in \mathscr{H}\right\}
$$

is bounded. We conclude that condition (b2) is also fulfilled.

Concerning condition (b3) we first note that by Lemma 12

$$
N\left(f\left(C^{2}\right)\right)=N\left(\left|f\left(C^{2}\right)\right|\right) \geq K N(|f(C)|)=K N(f(C))
$$


holds for every $C \in \mathscr{A}_{+}^{-1}$. Now, selecting any $X \in \mathscr{A}_{+}^{-1}$ and setting $Y=$ $X^{1 / 2} B^{-1} X^{1 / 2}$ we easily deduce that

$$
\begin{gathered}
d_{N, f}(X, B \diamond X)=N\left(f\left(X^{1 / 2}\left(B X^{-1} B\right)^{-1} X^{1 / 2}\right)\right) \\
=N\left(f\left(X^{1 / 2} B^{-1} X B^{-1} X^{1 / 2}\right)\right)=N\left(f\left(Y^{2}\right)\right) \geq K N(f(Y)) \\
=K N\left(f\left(X^{1 / 2} B^{-1} X^{1 / 2}\right)\right)=K d_{N, f}(X, B) .
\end{gathered}
$$

This means that condition (b3) is also satisfied. Therefore, all assumptions (b1)(b3) are fulfilled for any pair $A, B \in \mathscr{A}_{+}^{-1}$.

Let us show now that for the sequence $\left(X_{n}\right)$ in $\mathscr{A}_{+}^{-1}$ and element $X \in \mathscr{A}_{+}^{-1}$ we have the convergence $X_{n} \rightarrow X$ in the operator norm topology if and only if $d_{N, f}\left(X, X_{n}\right) \rightarrow 0$. To see this, assume $X_{n} \rightarrow X$ in the operator norm topology. Then $X^{1 / 2} X_{n}^{-1} X^{1 / 2} \rightarrow I$ which implies $f\left(X^{1 / 2} X_{n}^{-1} X^{1 / 2}\right) \rightarrow f(I)=0$. By the equivalence of $N$ to the operator norm, we obtain

$$
d_{N, f}\left(X, X_{n}\right)=N\left(f\left(X^{1 / 2} X_{n}^{-1} X^{1 / 2}\right)\right) \rightarrow 0 .
$$

Conversely, if the above convergence holds, then we have

$$
f\left(X^{1 / 2} X_{n}^{-1} X^{1 / 2}\right) \rightarrow 0
$$

in the operator norm. By the continuity of $f$ and the property (c1), it is easy to verify that we necessarily have

$$
X^{1 / 2} X_{n}^{-1} X^{1 / 2} \rightarrow I
$$

which implies that $X_{n} \rightarrow X$ in the operator norm.

Remark 14. In the above proposition we have supposed that the complete symmetric norm $N$ on $\mathscr{A}$ satisfies $N(|A|)=N(A)$ for all $A \in \mathscr{A}$. We do not know if it is really necessary to assume this or any complete symmetric norm automatically has this property. Nevertheless we suspect a negative answer.

Proof of Theorem 4. By Proposition 13 the conditions (b1)-(b3) are satisfied in Theorem 3 for all $A, B \in \mathscr{A}_{+}^{-1}$. The argument used there to verify (b1) shows that (b4) is fulfilled, too. Therefore, the conclusion in Theorem 3 holds for any pair $A, B \in \mathscr{A}_{+}^{-1}$ which gives us that the transformation $\phi$ is an inverted Jordan triple isomorphism. Its continuity follows from the last statement in Proposition 13 which is valid for the generalized distance measure $d_{M, g}$, too.

In accordance with our original plan, the next step we make is to prove Theorem 5 which describes the structure of continuous Jordan triple isomorphisms between the positive definite cones of von Neumann factors. Our idea how to do it comes from the paper [23]. Namely, we first verify that any such map is automatically Lipschitz in a small neighborhood of the identity $I$. This is the content of the next lemma. Its proof relies on some appropriate modifications in the proof of Lemma 5 in [23].

Lemma 15. Let $\mathscr{A}, \mathscr{B}$ be $C^{*}$-algebras. Let $\phi: \mathscr{A}_{+}^{-1} \rightarrow \mathscr{B}_{+}^{-1}$ be a continuous Jordan triple map. Then $\phi$ is a Lipschitz function in a neighborhood of the identity. 
Proof. We begin with the following important observation. For any $A \in \mathscr{A}_{+}^{-1}$ which is close enough to $I$ we have

$$
\frac{1}{2}\|A-I\| \leq\|\log A\| \leq 2\|A-I\| .
$$

Indeed, this follows easily from the inequalities

$$
\left\|e^{H}-I\right\| \leq e^{\|H\|}-1, \quad H \in \mathscr{A}_{s},
$$

and

$$
\|\log A\| \leq-\log (1-\|A-I\|), \quad A \in \mathscr{A}_{+}^{-1} \text { with }\|A-I\|<1,
$$

and from elementary properties of the exponential and logarithm functions of a real variable.

For temporary use, let $\mathscr{G}_{r}$ denote the closed ball in $\mathscr{A}_{+}^{-1}$ with center $I$ and radius $0<r<1$. We assert that there exists an $r$ with $0<r<1$ and another positive number $L$ for which $\|\phi(A)-I\| \leq L\|A-I\|$ holds for all $A \in \mathscr{G}_{r}$. Assume on the contrary that there is a sequence $\left(A_{k}\right)$ of elements of $\mathscr{A}_{+}^{-1}$ such that

$$
\left\|A_{k}-I\right\|<1 / k \text { and }\left\|\phi\left(A_{k}\right)-I\right\|>k\left\|A_{k}-I\right\|
$$

hold for every $k \in \mathbb{N}$. Since $A_{k} \rightarrow I$, it follows that $\phi\left(A_{k}\right) \rightarrow \phi(I)=I$, too. (Observe that $\phi(I)^{3}=\phi\left(I^{3}\right)=\phi(I)$ implies $\phi(I)=I$.) Clearly, we have

$$
\left\|\phi\left(A_{k}\right)-I\right\|=\epsilon_{k}, \quad \epsilon_{k}<1
$$

for large enough $k \in \mathbb{N}$ and in what follows we consider only such indexes $k$. Choose positive integers $l_{k}$ such that

$$
1 /\left(l_{k}+1\right) \leq \epsilon_{k}<1 / l_{k} \text {. }
$$

By (15), for large enough $k$ we have $\left\|\log A_{k}\right\| \leq 2\left\|A_{k}-I\right\|$ and hence obtain

$$
\left\|\log A_{k}^{l_{k}}\right\|=l_{k}\left\|\log A_{k}\right\| \leq 2 l_{k}\left\|A_{k}-I\right\|<2 l_{k}\left(\epsilon_{k} / k\right)<2 / k \rightarrow 0
$$

as $k \rightarrow \infty$. It follows that $A_{k}^{l_{k}} \rightarrow I$ and hence $A_{k}^{l_{k}+1} \rightarrow I$. Therefore, we infer $\phi\left(A_{k}^{l_{k}+1}\right) \rightarrow \phi(I)=I$. However, using (15) again, for large enough $k$ we also have

$$
\begin{gathered}
\frac{1}{2} \leq \frac{\epsilon_{k}\left(l_{k}+1\right)}{2}=\frac{l_{k}+1}{2}\left\|\phi\left(A_{k}\right)-I\right\| \leq \\
\left(l_{k}+1\right)\left\|\log \phi\left(A_{k}\right)\right\|=\left\|\log \phi\left(A_{k}^{l_{k}+1}\right)\right\| \rightarrow 0
\end{gathered}
$$

which is a contradiction. Consequently, there do exist positive real numbers $r(<1)$ and $L$ such that $\|\phi(A)-I\| \leq L\|A-I\|$ holds for all $A \in \mathscr{G}_{r}$. Clearly, $\phi$ is necessarily bounded on $\mathscr{G}_{r}$.

To complete the proof, let $s$ be a not yet specified positive number with $s<r$ and pick arbitrary $C, D \in \mathscr{G}_{s}$. Let $B=\sqrt{C}$ and $A=B^{-1} D B^{-1}$. Considering the inequality

$$
\begin{gathered}
\|A-I\|=\left\|B^{-1} D B^{-1}-I\right\| \\
\leq\left\|B^{-1}-I\right\|\|D\|\left\|B^{-1}\right\|+\|D-I\|\left\|B^{-1}\right\|+\left\|B^{-1}-I\right\|
\end{gathered}
$$


we see that choosing small enough $s>0$ we have $\|A-I\|<r$ and $\|B-I\|<r$. Assuming $C \neq D$ we can compute

$$
\begin{gathered}
\frac{\|\phi(D)-\phi(C)\|}{\|D-C\|}=\frac{\left\|\phi(B) \phi(A) \phi(B)-\phi(B)^{2}\right\|}{\left\|B A B-B^{2}\right\|} \leq \\
\frac{\left\|B^{-1}\right\|^{2}\|\phi(B)\|^{2}\|\phi(A)-I\|}{\|A-I\|}=\left\|C^{-1}\right\|\|\phi(C)\| \frac{\|\phi(A)-I\|}{\|A-I\|} \\
\leq L\left\|C^{-1}\right\|\|\phi(C)\| .
\end{gathered}
$$

Clearly, the function $C \mapsto\left\|C^{-1}\right\|\|\phi(C)\|$ is bounded on $\mathscr{G}_{s}$ (recall that $s<r$ ) and thus we obtain the desired Lipschitz property of $\phi$ in a neighborhood of $I$.

The next lemma shows that every continuous Jordan triple map from $\mathscr{A}_{+}^{-1}$ into $\mathscr{B}_{+}^{-1}$ is the exponential of a commutativity preserving linear map from $\mathscr{A}_{s}$ to $\mathscr{B}_{s}$ composed by the logarithmic function. Similarly to the case of matrix algebras treated in [23], this plays an essential role in the proof of Theorem 5. We say that a linear transformation $f: \mathscr{A}_{s} \rightarrow \mathscr{B}_{s}$ preserves commutativity (more precisely preserves commutativity in one direction) if for any pair $T, S \in \mathscr{A}_{S}$ of commuting elements we have that $f(T), f(S) \in \mathscr{B}_{S}$ commute, too. The proof of the next lemma follows the proof of Lemma 6 in [23] (presented for matrices) except its last paragraph.

Lemma 16. Let $\mathscr{A}, \mathscr{B}$ be $C^{*}$-algebras. Assume $\phi: \mathscr{A}_{+}^{-1} \rightarrow \mathscr{B}_{+}^{-1}$ is a continuous Jordan triple map. Then there exists a commutativity preserving linear transformation $f: \mathscr{A}_{S} \rightarrow \mathscr{B}_{s}$ such that

$$
\phi(A)=e^{f(\log A)}, \quad A \in \mathscr{A}_{+}^{-1} .
$$

Proof. We define $f: \mathscr{A}_{s} \rightarrow \mathscr{B}_{s}$ by

$$
f(T)=\log \phi\left(e^{T}\right), \quad T \in \mathscr{A}_{s} .
$$

We clearly have (17) and need only to show that $f$ is linear and preserves commutativity.

Pick arbitrary $A \in \mathscr{A}_{+}^{-1}$. Observe that since $\phi$ is a Jordan triple map, we have

$$
\phi\left(A^{n}\right)=\phi(A)^{n}
$$

for all integers $n=0,1,2, \ldots$. This easily implies that $\phi\left(A^{1 / n}\right)=\phi(A)^{1 / n}$. From $\phi(A) \phi\left(A^{-2}\right) \phi(A)=\phi(I)=I$ we have $\phi\left(A^{-1}\right)^{2}=\left(\phi(A)^{-1}\right)^{2}$ implying that $\phi$ preserves the inverse operation and hence (18) holds for all integers $n$. Therefore, $\phi\left(A^{r}\right)=\phi(A)^{r}$ is valid for all $A \in \mathscr{A}_{+}^{-1}$ and rational number $r$. By the continuity of $\phi$ we obtain that $\phi\left(A^{t}\right)=\phi(A)^{t}$ is true for every real number $t$, too. This obviously implies that $f$ is homogeneous. 
We next prove that $f: \mathscr{A}_{s} \rightarrow \mathscr{B}_{s}$ is additive. Pick $T, S, H \in \mathscr{A}_{s}$. We compute

$$
\begin{gathered}
\frac{e^{(t / 2) T} e^{t S} e^{(t / 2) T}-e^{t H}}{t}= \\
\frac{\left(e^{(t / 2) T}-I\right) e^{t S} e^{(t / 2) T}+\left(e^{t S}-I\right) e^{(t / 2) T}+\left(e^{(t / 2) T}-I\right)-\left(e^{t H}-I\right)}{t} \rightarrow \\
T / 2+S+T / 2-H=T+S-H
\end{gathered}
$$

as $t \rightarrow 0$. It follows that

$$
\lim _{t \rightarrow 0} \frac{e^{(t / 2) T} e^{t S} e^{(t / 2) T}-e^{t H}}{t}=0 \Longleftrightarrow H=T+S .
$$

If $H=T+S$, then using (17) and the Lipschitz property of $\phi$ in a neighborhood of $I$ that has been proven in Lemma 15 we have

$$
\begin{gathered}
\frac{e^{(t / 2) f(T)} e^{t f(S)} e^{(t / 2) f(T)}-e^{t f(H)}}{t}= \\
\frac{\phi\left(e^{(t / 2) T}\right) \phi\left(e^{t S}\right) \phi\left(e^{(t / 2) T}\right)-\phi\left(e^{t H}\right)}{t}= \\
\frac{\phi\left(e^{(t / 2) T} e^{t S} e^{(t / 2) T}\right)-\phi\left(e^{t H}\right)}{t} \rightarrow 0
\end{gathered}
$$

as $t \rightarrow 0$. On the other hand, as in (19) we infer

$$
\frac{e^{(t / 2) f(T)} e^{t f(S)} e^{(t / 2) f(T)}-e^{t f(H)}}{t} \rightarrow f(T)+f(S)-f(H) .
$$

This gives us that $f(T)+f(S)-f(T+S)=0$, i.e., $f$ is additive.

To verify the commutativity preserving property of $f$ first observe that we have

$$
\phi(\sqrt{A} B \sqrt{A})=\phi(\sqrt{A}) \phi(B) \phi(\sqrt{A})=\sqrt{\phi(A)} \phi(B) \sqrt{\phi(A)}
$$

for every $A, B \in \mathscr{A}_{+}^{-1}$. We now recall the following notion and fact. Given positive elements $D, F$ of a unital $C^{*}$-algebra define their so-called sequential product by $\sqrt{D} F \sqrt{D}$. It is an interesting fact that commutativity of $D, F$ with respect to this product is equivalent to the commutativity of $D, F$ with respect to the usual product. A short proof of this fact has been given in Proposition 1 in [2]. It is then clear that $\phi$ preserves commutativity which apparently implies the commutativity preserving property of $f$, too.

The next lemma what we shall need for the proof of Theorem 5 provides a characterization of tracial continuous linear functionals on von Neumann algebras in terms of their behavior with respect to the Jordan triple product. It says that the continuous linear functional $l$ which is real valued on self-adjoint elements is tracial if and only if the (non-linear) functional exp $\circ l \circ \log$ is a Jordan triple map on the positive definite cone.

Lemma 17. Let $\mathscr{A}$ be a von Neumann algebra and $l: \mathscr{A} \rightarrow \mathbb{C}$ a continuous linear functional which has real values on $\mathscr{A}_{s}$. Then l satisfies

$$
l(\log A B A)=l(\log A)+l(\log B)+l(\log A), \quad A, B \in \mathscr{A}_{+}^{-1} .
$$


if and only ifl is tracial.

Proof. Assume that $l$ satisfies (20). We first follow an argument similar to the one given in the proof of Theorem 2 in [20]. Pick projections $P, Q$ in $\mathscr{A}$. Let

$$
A=I+t P, \quad B=I+t Q,
$$

where $t>-1$ is any real number. Easy computation shows that

$$
\begin{gathered}
A B A=(I+t P)(I+t Q)(I+t P) \\
=I+t(2 P+Q)+t^{2}(P+P Q+Q P)+t^{3}(P Q P) .
\end{gathered}
$$

Recall that in an arbitrary unital Banach algebra, for any element $a$ with $\|a\|<1$ we have

$$
\log (1+a)=\sum_{n=1}^{\infty} \frac{(-1)^{n+1} a^{n}}{n} .
$$

This shows that for a suitable positive $\epsilon$, the elements $\log (A B A), \log A, \log B$ of $\mathscr{A}$ can be expressed by power series of $t(|t|<\epsilon)$ with algebra coefficients. In particular, considering the coefficients of $t^{3}$ on both sides of the equality (20) and using their uniqueness, we obtain the following equation

$$
\begin{gathered}
l\left(P Q P-\frac{1}{2}((2 P+Q)(P+P Q+Q P)\right. \\
\left.+(P+P Q+Q P)(2 P+Q))+\frac{1}{3}(2 P+Q)^{3}\right) \\
=l\left(\frac{1}{3}(P+Q+P)\right) .
\end{gathered}
$$

Executing the operations and subtracting those terms which appear on both sides of this equation, we arrive at the following equality

$$
l\left(\frac{1}{3}(P Q P)-\frac{1}{3}(Q P Q)\right)=0 .
$$

Therefore,

$$
l(P Q P)=l(Q P Q)
$$

holds for all projections $P, Q \in \mathscr{A}$. We assert that this implies that $l$ is tracial, i.e., $l(X Y)=l(Y X), X, Y \in \mathscr{A}$. To verify this, we apply an idea from the proof of Lemma 1 in [4]. Namely, select an arbitrary pair $P, Q$ of projections in $\mathscr{A}$, define $S=I-2 P$ and compute

$$
\begin{aligned}
l(Q+S Q S) & =\frac{1}{2} l((I-S) Q(I-S)+(I+S) Q(I+S)) \\
= & \frac{1}{2} l(4 P Q P+4(I-P) Q(I-P)) \\
= & 2 l(P Q P+(I-P) Q(I-P)) \\
= & 2 l(Q P Q+Q(I-P) Q)=2 l(Q) .
\end{aligned}
$$

Since the symmetries (i.e., self-adjoint unitaries) in $\mathscr{A}$ are exactly the elements of the form $S=I-2 P$ with some projection $P \in \mathscr{A}$, we obtain that $l(Q)=l(S Q S)$ holds for every symmetry $S$ and every projection $Q$ in $\mathscr{A}$. By the continuity of 
the linear functional $l$ and using spectral theorem, we infer $l(X)=l(S X S)$ holds for any $X \in \mathscr{A}$ and symmetry $S \in \mathscr{A}$. This implies that

$$
l(S X)=l(S(X S) S)=l(X S)
$$

for all $X \in \mathscr{A}$ and symmetry $S \in \mathscr{A}$. Plainly, this gives us that $l(P X)=l(X P)$ holds for every projection $P \in \mathscr{A}$. Finally, we conclude that $l(X Y)=l(Y X)$ for all $X, Y \in \mathscr{A}$.

Conversely, if $l: \mathscr{A} \rightarrow \mathbb{C}$ is a continuous linear functional which has real values on $\mathscr{A}_{s}$ and tracial, we need to prove that

$$
e^{l(\log A B A)}=e^{l(\log A)+l(\log B)+l(\log A)}
$$

holds for all $A, B \in \mathscr{A}_{+}^{-1}$. This can be proven following the proof of Lemma 2 and Lemma 3 in [9].

In the proofs of our theorems on the structures of continuous Jordan triple isomorphisms we shall also need a particular case of the following result which will appear in [24].

Proposition 18. Let $\mathscr{A}$ be a $C^{*}$-algebra. If $c \notin\{-1,0,1\}$ is a real number with the property that for any pair $A, B \in \mathscr{A}_{+}^{-1}$ we have a real number $\lambda$ such that

$$
(A B A)^{c}=\lambda A^{c} B^{c} A^{c},
$$

then the algebra $\mathscr{A}$ is commutative. Similarly, if $m$ is an integer $m \notin\{-1,0,1\}$ and for any pair $U, V \in \mathscr{A}_{u}$ we have a scalar $\mu$ such that

$$
(U V U)^{m}=\mu U^{m} V^{m} U^{m},
$$

then $\mathscr{A}$ is commutative.

We now have all preliminary information to present the proof of Theorem 5.

Proof of Theorem 5. Let $\phi: \mathscr{A}_{+}^{-1} \rightarrow \mathscr{B}_{+}^{-1}$ be a continuous Jordan triple isomorphism. Applying Lemma 16 we have a bijective linear transformation $f: \mathscr{A}_{s} \rightarrow$ $\mathscr{B}_{s}$ such that $\phi(A)=\exp f(\log A), A \in \mathscr{A}_{+}^{-1}$ and $f$ preserves commutativity. Since $\phi^{-1}$ is also a Jordan triple isomorphism, it follows from the last part of the proof of Lemma 16 that $\phi^{-1}$ also preserves commutativity implying that $f$ preserves commutativity in both directions. It follows that $\mathscr{B}$ is a factor von Neumann algebra, too.

If $\mathscr{A}$ is of type $\mathrm{I}_{1}$, then so is $\mathscr{B}$ and the theorem reduces to the description of all continuous multiplicative bijections of the positive real line. These maps are well-known to be exactly the power functions corresponding to nonzero exponents. So, in this case the assertion is trivial and hence in what follows we assume that $\mathscr{A}$ is not of type $\mathrm{I}_{1}$ and not of type $\mathrm{I}_{2}$.

We extend $f$ from $\mathscr{A}_{s}$ to a linear transformation onto $\mathscr{A}$ in the following trivial way:

$$
F(A+i B)=f(A)+i f(B) \quad\left(A, B \in \mathscr{A}_{S}\right) .
$$

Clearly, $F: \mathscr{A} \rightarrow \mathscr{B}$ is a bijective linear transformation. Since an operator is normal if and only if its real and imaginary parts commute, we see that $F$ sends the normal elements of $\mathscr{A}$ to normal elements of $\mathscr{B}$. There are structural results 
concerning such maps. We refer to Theorem 4.1 in [6] on the form of normal preserving linear transformations between centrally closed prime algebras satisfying some additional conditions which can be applied here (see the introduction of that paper for the explanation of the necessary concepts). We obtain that there is a nonzero complex number $c$, an algebra ${ }^{*}$-isomorphism or an algebra *-antiisomorphism $\theta: \mathscr{A} \rightarrow \mathscr{B}$, and a linear functional $l: \mathscr{A} \rightarrow \mathbb{C}$ such that

$$
F(A)=c \theta(A)+l(A) I \quad(A \in \mathscr{A}) .
$$

We claim that $c$ is real and $l$ maps $\mathscr{A}_{s}$ into $\mathbb{R}$. In order to see this, let $P$ be a nontrivial projection in $\mathscr{A}$. Then $c \theta(P)+l(P) I$ is a self-adjoint element of $\mathscr{B}$ and $\theta(P)$ is a nontrivial projection. This easily gives us first that $l(P)$ and then that $c$ are real numbers. Next, for any $A \in \mathscr{A}_{s}$ we have that $c \theta(A)+l(A) I$ and $c \theta(A)$ are both self-adjoint implying that $l(A) \in \mathbb{R}$.

Clearly, $c$ is not zero. We have

$$
\phi(A)=e^{c \theta(\log A)+l(\log A) I}=e^{l(\log A)} \theta\left(A^{c}\right), \quad A \in \mathscr{A}_{+}^{-1} .
$$

Since $\phi$ is a Jordan triple isomorphism from $\mathscr{A}_{+}^{-1}$ onto $\mathscr{B}_{+}^{-1}$ and $\theta$ is an algebra *isomorphism or an algebra *-antiisomorphism from $\mathscr{A}$ onto $\mathscr{B}$, it readily follows that

$$
e^{l(\log A B A)} \theta\left((A B A)^{c}\right)=e^{l(\log A)+l(\log B)+l(\log A)} \theta\left(A^{c} B^{c} A^{c}\right), \quad A, B \in \mathscr{A}_{+}^{-1}
$$

from which we obtain

$$
e^{l(\log A B A)}(A B A)^{c}=e^{l(\log A)+l(\log B)+l(\log A)} A^{c} B^{c} A^{c}, \quad A, B \in \mathscr{A}_{+}^{-1} .
$$

In particular, $(A B A)^{c}$ and $A^{c} B^{c} A^{c}$ are scalar multiples of each other for all $A, B \in$ $\mathscr{A}_{+}^{-1}$. By Proposition 18 it follows that $c$ is either 1 or -1 . Therefore, we have $e^{l(\log A B A)}=e^{l(\log A)+l(\log B)+l(\log A)}$ implying

$$
l(\log A B A)=l(\log A)+l(\log B)+l(\log A), \quad A, B \in \mathscr{A}_{+}^{-1} .
$$

By the continuity of $\phi$ and using the fact that $\theta$ is necessarily isometric, we have that $l$ is a continuous linear functional. Applying Lemma 17 we have that $l$ is tracial. By the injectivity of $\phi$ we have $\phi(e I) \neq \phi(I)=I$ which gives $l(I)+c \neq 0$. This proves the necessity part of our theorem.

Conversely, if $l: \mathscr{A} \rightarrow \mathbb{C}$ is a continuous linear functional which is real valued on $\mathscr{A}_{s}$ and tracial, then by Lemma 17

$$
e^{l(\log A B A)}=e^{l(\log A)+l(\log B)+l(\log A)}
$$

holds for all $A, B \in \mathscr{A}_{+}^{-1}$. If $c \in\{-1,1\}$ and $l(I) \neq-c$ is also true, then one can readily verify that for any algebra ${ }^{*}$-isomorphism or algebra ${ }^{*}$-antiisomorphism $\theta: \mathscr{A} \rightarrow \mathscr{B}$, the map $\phi: \mathscr{A}_{+}^{-1} \rightarrow \mathscr{B}_{+}^{-1}$ defined by

$$
\phi(A)=e^{l(\log A)} \theta\left(A^{c}\right), \quad A \in \mathscr{A}_{+}^{-1}
$$

is a continuous Jordan triple isomorphism.

After this the proof of Theorem 6 is very simple. We note that on a von Neumann algebra $\mathscr{A}$ any symmetric norm $N$ has the property $N(|A|)=N(A), A \in \mathscr{A}$ 
which follows easily from the fact that the components of the polar decomposition of any element in $\mathscr{A}$ belong to $\mathscr{A}$.

Proof of Theorem 6. Let $\phi: \mathscr{A}_{+}^{-1} \rightarrow \mathscr{B}_{+}^{-1}$ be a surjective function which satisfies

$$
d_{M, g}(\phi(A), \phi(B))=d_{N, f}(A, B), \quad A, B \in \mathscr{A}_{+}^{-1} .
$$

From Theorem 4 we obtain that $\phi$ is a continuous inverted Jordan triple isomorphism. As mentioned in between the formulations of Theorems 4 and 5, the map $\psi()=.\phi(I)^{-1 / 2} \phi(.) \phi(I)^{-1 / 2}$ is a continuous Jordan triple isomorphism and hence the latter result applies and we obtain the form (5). As for the last statement in the theorem on the disappearance of $l$ in the case of infinite factors, we refer the remark given after Theorem 5 . The proof is complete.

To see cases where the tracial linear functional in (5) really shows up we refer to Theorem 3 in [23].

Remark 19. We present a sort of application of Theorems 4 and 6 . In the paper [15] Honma and Nogawa considered metrics on the positive definite cone of a $C^{*}$-algebra $\mathscr{A}$ of the form

$$
d_{\alpha}(A, B)=\left\|\log \left(A^{-\alpha / 2} B^{\alpha} A^{-\alpha / 2}\right)^{1 / \alpha}\right\|, \quad A, B \in \mathscr{A}_{+}^{-1},
$$

where $\alpha$ is a given nonzero real number. They described the structure of surjective isometries between two such spaces.

Observe that the problem can also be treated in the framework that we have presented above. Indeed, let $\mathscr{A}, \mathscr{B}$ be $C^{*}$-algebras, $\alpha, \beta$ given nonzero real numbers and $\phi: \mathscr{A}_{+}^{-1} \rightarrow \mathscr{B}_{+}^{-1}$ a surjective map which is an isometry with respect to the pair $d_{\alpha}, d_{\beta}$ of metrics, i.e., which satisfies

$$
\left\|\log \left(\phi(A)^{-\beta / 2} \phi(B)^{\beta} \phi(A)^{-\beta / 2}\right)^{1 / \beta}\right\|=\left\|\log \left(A^{-\alpha / 2} B^{\alpha} A^{-\alpha / 2}\right)^{1 / \alpha}\right\|
$$

for all $A, B \in \mathscr{A}_{+}^{-1}$. Define $\psi(A)=\phi\left(A^{1 / a}\right)^{\beta}, A \in \mathscr{A}_{+}^{-1}$. Hence, $\psi: \mathscr{A}_{+}^{-1} \rightarrow \mathscr{B}_{+}^{-1}$ is a bijective map for which

$$
(1 /|\beta|)\left\|\log \left(\psi(A)^{-1 / 2} \psi(B) \psi(A)^{-1 / 2}\right)\right\|=(1 /|\alpha|)\left\|\log \left(A^{-1 / 2} B A^{-1 / 2}\right)\right\|
$$

holds for all $A, B \in \mathscr{A}_{+}^{-1}$. Now, apply Theorem 4 for $N()=.(1 /|\beta|)\|\|,. f(y)=$ $-\log y, y>0$ and $M()=.(1 /|\alpha|)\|\|,. g(y)=-\log y, y>0$. We infer that $\psi: \mathscr{A}_{+}^{-1} \rightarrow$ $\mathscr{B}_{+}^{-1}$ is an inverted Jordan triple isomorphism. This means that

$$
\phi\left(A^{1 / \alpha}\right)^{\beta} \phi\left(B^{1 / \alpha}\right)^{-\beta} \phi\left(A^{1 / \alpha}\right)^{\beta}=\phi\left(\left(A B^{-1} A\right)^{1 / \alpha}\right)^{\beta}, \quad A, B \in \mathscr{A}_{+}^{-1} .
$$

Replacing here $A$ by $A^{\alpha}$ and $B$ by $B^{\alpha}$ we immediately get

$$
\phi(A)^{\beta} \phi(B)^{-\beta} \phi(A)^{\beta}=\phi\left(\left(A^{\alpha} B^{-\alpha} A^{\alpha}\right)^{1 / \alpha}\right)^{\beta}
$$

and hence that

$$
\left(\phi(A)^{\beta} \phi(B)^{-\beta} \phi(A)^{\beta}\right)^{1 / \beta}=\phi\left(\left(A^{\alpha} B^{-\alpha} A^{\alpha}\right)^{1 / \alpha}\right), \quad A, B \in \mathscr{A}_{+}^{-1} .
$$

This is just the conclusion formulated in Proposition 2 in [15] on the algebraic behavior of the isometries with respect to the pair $d_{\alpha}, d_{\beta}$ of metrics which statement plays important role in that paper. Using the same ideas and applying Theorem 6 it should not be difficult to derive Corollary 9 in [15]. Furthermore, 
observe that by our general results one can treat the cases of more general distances. Indeed, in [15] also metrics on the set of all positive definite matrices of the form

$$
d_{\|\cdot\|, \alpha, \alpha}(A, B)=\left\|\log \left(A^{-\alpha / 2} B^{\alpha} A^{-\alpha / 2}\right)^{1 / \alpha}\right\|
$$

where $\|$.$\| \| is a unitarily invariant norm on \mathbb{M}_{n}$ have been mentioned but structural result on the corresponding isometries was not obtained. Clearly, our approach above applies also in that situation and using it one can easily describe the corresponding isometries. We omit the details.

We now turn to the proofs of our results on transformations between unitary groups which respect a pair of generalized distance measures. Our approach is quite similar to the one we have followed in the case of the positive definite cone. We first present an appropriate general Mazur-Ulam type result, then show that under certain conditions the surjective "generalized isometries" what we consider are continuous inverted Jordan triple isomorphisms. Next we describe the structure of those isomorphisms between von Neumann factors and finally, after gathering the necessary information, we prove our result on the structure of surjective "generalized isometries" beween unitary groups of von Neumann factors.

The general Mazur-Ulam type theorem what we need here reads as follows.

Proposition 20. Suppose that $G$ and $H$ are groups equipped with generalized distance measures $d$ and $\rho$, respectively. Pick $a, b \in G$, set

$$
L_{a, b}=\left\{x \in G: d(a, x)=d\left(x, b a^{-1} b\right)=d(a, b)\right\},
$$

and assume the following:

(e1) $d\left(b x^{-1} b, b x^{\prime-1} b\right)=d\left(x^{\prime}, x\right)$ holds for all $x, x^{\prime} \in G$;

(e2) $\sup \left\{d(x, b): x \in L_{a, b}\right\}<\infty$;

(e3) there exists a constant $K>1$ such that

$$
d\left(x, b x^{-1} b\right) \geq K d(x, b), \quad x \in L_{a, b} ;
$$

(e4) $\rho\left(c y^{-1} c^{\prime}, c y^{\prime-1} c^{\prime}\right)=\rho\left(y^{\prime}, y\right)$ holds for all $c, c^{\prime}, y, y^{\prime} \in H$.

Then for any surjective map $\phi: G \rightarrow H$ which satisfies

$$
\rho\left(\phi(x), \phi\left(x^{\prime}\right)\right)=d\left(x, x^{\prime}\right), \quad x, x^{\prime} \in G
$$

we have

$$
\phi\left(b a^{-1} b\right)=\phi(b) \phi(a)^{-1} \phi(b) .
$$

Proof. First observe that $\phi$ is also injective and hence bijective. Let $\varphi(x)=$ $b x^{-1} b, x \in G$, define $\psi(y)=\phi(a) y^{-1} \phi\left(b a^{-1} b\right), y \in H$ and let $T=\phi$. One can readily check that Proposition 11 applies and results in $\psi(\phi(b))=\phi(b)$. This immediately implies

$$
\phi\left(b a^{-1} b\right)=\phi(b) \phi(a)^{-1} \phi(b)
$$

and we are done.

We proceed with the proof of Theorem 7. 
Proof of Theorem 7. We apply Proposition 20 in the following setting: $G=\mathscr{A}_{u}$, $H=\mathscr{B}_{u}, d=d_{N, f}, \rho=d_{M, g}$. We observe that conditions (e1), (e2), (e4) are satisfied for all $A, B, X, X^{\prime} \in \mathscr{A}_{u}$ and $C, D, Y, Y^{\prime} \in \mathscr{B}_{u}$. Indeed, to see (e1) we can compute

$$
\begin{gathered}
f\left(B X^{-1} X^{\prime} B^{-1}\right)=B f\left(X^{-1} X^{\prime}\right) B^{-1} \\
=B f\left(X^{-1}\left(X^{\prime} X^{-1}\right) X\right) B^{-1}=B X^{-1} f\left(X^{\prime} X^{-1}\right) X B^{-1}
\end{gathered}
$$

and hence, by the unitary similarity invariance of $N$, it follows that

$$
d_{N, f}\left(B X^{-1} B^{\prime}, B X^{\prime-1} B^{\prime}\right)=d_{N, f}\left(X^{\prime}, X\right)
$$

holds for all $B, B^{\prime}, X, X^{\prime} \in \mathscr{A}_{u}$. As for (e2), the set of all values of $d_{N, f}(.,$.$) is$ bounded which is a consequence of the boundedness of $f$ and the equivalence of $N$ to the operator norm.

By (d2) we have that $N\left(f\left(U^{2}\right)\right) \geq K N(f(U))$ if $U \in \mathscr{A}_{u}$ is close enough (in the operator norm) to $I$. In what follows we show that (e3) is also satisfied provided $A, B \in \mathscr{A}_{u}$ are close enough to each other in the operator norm. We warn the reader that we are going to argue rather vaguely avoiding the precise " $\epsilon-\delta$ technique" which would make the proof much more lengthy. So, let $A, B \in \mathscr{A}_{u}$ be unitaries which are close to each other in the operator norm. Then picking $X$ from $L_{A, B}$, referring to the property (d1) of $f$ and the equivalence of $N$ to the operator norm, we have that $N\left(f\left(A X^{-1}\right)\right)=N\left(f\left(A B^{-1}\right)\right)$ is small. This gives us that $A X^{-1}$ is close to the identity, i.e., $X$ is close to $A$ in the operator norm. Hence we obtain that $X$ is close also to $B$ implying that $X B^{-1}$ is close to the identity in the operator norm. By (d2) we have

$$
d_{N, f}\left(X, B X^{-1} B\right)=N\left(f\left(\left(X B^{-1}\right)^{2}\right)\right) \geq K N\left(f\left(X B^{-1}\right)\right)=K d_{N, f}(X, B) .
$$

This shows that (e3) really holds for $A, B \in \mathscr{A}_{u}$ which are close enough to each other in the operator norm. The fact that (e4) is also valid follows from the argument we have presented relating to condition (e1) above.

Therefore, by Proposition 20 we have that for $A, B \in \mathscr{A}_{u}$ which are close enough to each other in the operator norm, the equality

$$
\phi\left(B A^{-1} B\right)=\phi(B) \phi(A)^{-1} \phi(B)
$$

holds. We can now follow the argument presented in the proof of Theorem 8 in [13] to conclude that from the validity of that equality for close enough $A, B$ we obtain that it necessarily holds globally, i.e., for all $A, B \in \mathscr{A}_{u}$, too. This completes the proof.

As we have mentioned after the formulation of Theorem 7, multiplying any inverted Jordan triple isomorphism $\phi$ between unitary groups by the element $\phi(I)^{-1}$ we obtain a Jordan triple isomorphism. Our next aim is to determine the structure of the continuous Jordan triple isomorphisms between unitary groups of von Neumann factors. As in the case of the positive definite cone, the first step is to show that any such map has Lipschitz property which in fact holds globally in the present situation. 
Lemma 21. Let $\mathscr{A}, \mathscr{B}$ be von Neumann algebras and $\phi: \mathscr{A}_{u} \rightarrow \mathscr{B}_{u}$ a continuous Jordan triple map, i.e., assume that

$$
\phi(U V U)=\phi(U) \phi(V) \phi(U), \quad U, V \in \mathscr{A}_{u} .
$$

Then $\phi$ is a Lipschitz function.

Proof. Since $\phi$ is a Jordan triple map, it follows that $\phi(I)^{3}=\phi(I)$, hence $\phi(I)^{2}=I$ which means that $\phi(I)$ is a symmetry (i.e., self-adjoint unitary). From $\phi(I) \phi(V) \phi(I)=\phi(V)$ we obtain $\phi(I) \phi(V)=\phi(V) \phi(I)$ for any $V \in \mathscr{A}_{u}$ which means that $\phi(I)$ commutes with the range of $\phi$. It follows easily that $\phi(I)^{-1} \phi($.) is also a Jordan triple map. Therefore, we may and do assume that $\phi(I)=I$. It is easy to see that in that case we have $\phi\left(U^{m}\right)=\phi(U)^{m}$ for any positive integer $m$ and $U \in \mathscr{A}_{u}$.

Next we follow the argument given in the proof of Lemma 6 in [22] but we need to make some necessary modifications due to the fact that here we consider operator algebras, not matrix algebras. We first assert that there exist positive real numbers $r, L$ such that $\|\phi(U)-I\| \leq L\|U-I\|$ holds for all $U \in \mathscr{A}_{u}$ with $\|U-I\|<r$. Assume on the contrary that we have a sequence $\left(U_{k}\right)$ in $\mathscr{A}_{u}$ such that $\left\|U_{k}-I\right\|<1 / k$ and

$$
\left\|\phi\left(U_{k}\right)-I\right\|>k\left\|U_{k}-I\right\|
$$

holds for every $k \in \mathbb{N}$. We have $U_{k} \rightarrow I$ and hence $\phi\left(U_{k}\right) \rightarrow I$ as $k \rightarrow \infty$. Denoting $\epsilon_{k}=\left\|\phi\left(U_{k}\right)-I\right\|$ we obviously have $\epsilon_{k}<1 / 2$ for large enough $k$. Choose positive integers $l_{k}$ such that

$$
1 /\left(l_{k}+1\right) \leq \epsilon_{k}<1 / l_{k}
$$

By (21) we have

$$
\epsilon_{k} / k>\left\|U_{k}-I\right\|
$$

and hence

$$
\left\|U_{k}^{l_{k}}-I\right\| \leq\left\|U_{k}-I\right\|\left\|U_{k}^{l_{k}-1}+\ldots+I\right\| \leq l_{k}\left\|U_{k}-I\right\|<\left(l_{k} \epsilon_{k}\right) / k<1 / k .
$$

It follows that $U_{k}^{l_{k}+1} \rightarrow I$ and we infer $\phi\left(U_{k}^{l_{k}+1}\right)=\phi\left(U_{k}\right)^{l_{k}+1} \rightarrow I$.

We shall need the following simple observation. Let $\lambda$ be a complex number of modulus 1 in the upper half plane. Clearly, the length of arc from 1 to $\lambda$ divided by the length of the corresponding chord is less than $\pi / 2$. Geometrical considerations show that assuming $n$ is a positive integer such that $n(\pi / 2)|\lambda-1|<\pi$, we necessarily have $n|\lambda-1|<(\pi / 2)\left|\lambda^{n}-1\right|$. It follows that for any unitary $V \in \mathscr{A}_{u}$ and positive integer $n$, the inequality $n\|V-I\|<2$ implies $n\|V-I\|<(\pi / 2)\left\|V^{n}-I\right\|$.

We have

$$
2 \epsilon_{k}\left(l_{k}+1\right)<2\left(l_{k}+1\right) / l_{k}<\pi,
$$

where in the last inequality we have used $l_{k} \geq 2$. This gives us that $\left(l_{k}+\right.$ 1) $\left\|\phi\left(U_{k}\right)-I\right\|<2$. Therefore, we compute

$$
1=\left(1 / \epsilon_{k}\right)\left\|\phi\left(U_{k}\right)-I\right\| \leq\left(l_{k}+1\right)\left\|\phi\left(U_{k}\right)-I\right\| \leq(\pi / 2)\left\|\phi\left(U_{k}\right)^{l_{k}+1}-I\right\| .
$$


But this clearly contradicts to the fact that $\phi\left(U_{k}\right)^{l_{k}+1} \rightarrow I$. Therefore, we do have positive real numbers $r, L$ such that $\|\phi(U)-I\| \leq L\|U-I\|$ holds for every $U \in \mathscr{A}_{u}$ with $\|U-I\|<r$. Since $\phi$ is bounded, we have (probably with another constant $L$ ) that $\|\phi(U)-I\| \leq L\|U-I\|$ is valid for every $U \in \mathscr{A}_{u}$.

To complete the proof, pick arbitrary unitaries $W, W^{\prime} \in \mathscr{A}_{u}$. Since every unitary in $\mathscr{A}$ is the exponential of a self-adjoint element multiplied by the imaginary unit $i$, we can choose $V \in \mathscr{A}_{u}$ such that $V^{2}=W^{\prime}$ and then find $U \in \mathscr{A}_{u}$ such that $V U V=W$. We compute

$$
\begin{gathered}
\quad\left\|\phi(W)-\phi\left(W^{\prime}\right)\right\|=\left\|\phi(V U V)-\phi\left(V^{2}\right)\right\| \\
=\left\|\phi(V) \phi(U) \phi(V)-\phi(V)^{2}\right\|=\|\phi(U)-I\| \\
\leq L\|U-I\|=L\left\|V U V-V^{2}\right\|=L\left\|W-W^{\prime}\right\| .
\end{gathered}
$$

This proves that $\phi$ is a Lipschitz function.

In the next lemma we show that every continuous Jordan triple map between unitary groups of von Neumann algebras gives rise to a certain commutativity preserving linear map between the self-adjoint parts of the underlying algebras. This result is parallel to Lemma 16. In the proof we follow the argument given in the proof of Lemma 7 in [22].

Lemma 22. Let $\mathscr{A}, \mathscr{B}$ be von Neumann algebras and $\phi: \mathscr{A}_{u} \rightarrow \mathscr{B}_{u}$ a continuous Jordan triple map. Then $\phi(I)$ is a symmetry which commutes with the range of $\phi$ and we have a commutativity preserving linear transformation $f: \mathscr{A}_{s} \rightarrow \mathscr{B}_{s}$ such that

$$
\phi\left(e^{i t A}\right)=\phi(I) e^{i t f(A)}, \quad t \in \mathbb{R}, A \in \mathscr{A}_{s} .
$$

Moreover, $f$ satisfies

$$
f(V A V)=\phi(V) f(A) \phi(V)
$$

for every $A \in \mathscr{A}_{s}$ and symmetry (self-adjoint unitary) $V \in \mathscr{A}_{u}$.

Proof. The assertion concerning $\phi(I)$ has been verified in the first part of the proof of Lemma 21. Just as there we can assume that $\phi$ is a unital Jordan triple map, $\phi(I)=I$. We have also learned that $\phi\left(V^{k}\right)=\phi(V)^{k}$ holds for every $V \in \mathscr{A}_{u}$ and positive integer $k$. We now show that $\phi$ preserves the inverse operation. To prove this, let $W \in \mathscr{A}_{u}$ be such that $W^{2}=V$. We compute

$$
\phi(W) \phi\left(V^{-1}\right) \phi(W)=\phi\left(W V^{-1} W\right)=\phi(I)=I
$$

which implies that

$$
\phi\left(V^{-1}\right)=\phi(W)^{-2}=\phi\left(W^{2}\right)^{-1}=\phi(V)^{-1} .
$$

It follows that $\phi\left(V^{k}\right)=\phi(V)^{k}$ holds for every integer $k$ and for every $V \in \mathscr{A}_{u}$. In the rest of the proof we shall use several times that, in particular, $\phi$ sends symmetries to symmetries.

In the next step we show that $\phi$ sends norm-continuous one-parameter unitary groups to norm-continuous one-parameter unitary groups. Pick an arbitrary self-adjoint element $T \in \mathscr{A}_{s}$ and define $S_{T}: \mathbb{R} \rightarrow \mathscr{B}_{u}$ by

$$
S_{T}(t)=\phi\left(e^{i t T}\right), \quad t \in \mathbb{R}
$$


We assert that $S_{T}$ is a norm-continuous one-parameter unitary group in $\mathscr{B}_{u}$. Since $\phi$ is continuous, $S_{T}$ is also continuous. We verify that $S_{T}\left(t+t^{\prime}\right)=$ $S_{T}(t) S_{T}\left(t^{\prime}\right)$ holds for every pair $t, t^{\prime}$ of real numbers. First select rational numbers $r$ and $r^{\prime}$ such that $r=\frac{k}{m}$ and $r^{\prime}=\frac{k^{\prime}}{m^{\prime}}$ with integers $k, k^{\prime}, m, m^{\prime}$. We compute

$$
\begin{aligned}
& S_{T}\left(r+r^{\prime}\right)=\phi\left(e^{i \frac{k m^{\prime}+k^{\prime} m}{m m^{\prime}} T}\right)=\phi\left(e^{i \frac{1}{m m^{\prime}} T}\right)^{k m^{\prime}+k^{\prime} m} \\
& =\phi\left(e^{i \frac{1}{m m^{\prime}} T}\right)^{k m^{\prime}} \phi\left(e^{i \frac{1}{m m^{\prime}} T}\right)^{k^{\prime} m}=S_{T}(r) S_{T}\left(r^{\prime}\right) .
\end{aligned}
$$

By the continuity of $S_{T}$, we deduce that $S_{T}\left(t+t^{\prime}\right)=S_{T}(t) S_{T}\left(t^{\prime}\right)$ holds for every pair $t, t^{\prime}$ of real numbers. By Stone's theorem we obtain that there exists a unique self-adjoint element $f(T) \in \mathscr{B}_{s}$, the generator of $S_{T}$, such that

$$
\phi\left(e^{i t T}\right)=S_{T}(t)=e^{i t f(T)}, \quad t \in \mathbb{R} .
$$

Observe that the generator belongs to $\mathscr{B}$ since in the present case (normcontinuous one-parameter unitary group) it can be obtained by differentiation, the limit of difference quotients taken in the norm topology.

We next prove that $f: \mathscr{A}_{s} \rightarrow \mathscr{B}_{S}$ is in fact a linear transformation. Pick $A, B, C \in$ $\mathscr{A}_{s}$. Similarly to the proof of Lemma 16 we compute

$$
=\frac{\frac{e^{i(t / 2) A} e^{i t B} e^{i(t / 2) A}-e^{i t C}}{i t}}{i t}
$$

as $t \rightarrow 0$. It follows that

$$
\lim _{t \rightarrow 0} \frac{e^{i(t / 2) A} e^{i t B} e^{i(t / 2) A}-e^{i t C}}{i t}=0 \Longleftrightarrow C=A+B .
$$

If $C=A+B$, then using the Lipschitz property of $\phi$ proven in Lemma 21 we have

$$
\begin{aligned}
& \frac{e^{i(t / 2) f(A)} e^{i t f(B)} e^{i(t / 2) f(A)}-e^{i t f(C)}}{i t} \\
= & \frac{\phi\left(e^{i(t / 2) A}\right) \phi\left(e^{i t B}\right) \phi\left(e^{i(t / 2) A}\right)-\phi\left(e^{i t C}\right)}{i t} \\
= & \frac{\phi\left(e^{i(t / 2) A} e^{i t B} e^{i(t / 2) A}\right)-\phi\left(e^{i t C}\right)}{i t} \rightarrow 0
\end{aligned}
$$

as $t \rightarrow 0$. On the other hand, just as in (22) above we have

$$
\frac{e^{i(t / 2) f(A)} e^{i t f(B)} e^{i(t / 2) f(A)}-e^{i t f(C)}}{i t} \rightarrow f(A)+f(B)-f(C) .
$$

This gives us that $f(A)+f(B)-f(A+B)=0$, i.e., $f$ is additive. The homogeneity of $f$ is trivial to see. Indeed, we have

$$
e^{i t \lambda f(A)}=\phi\left(e^{i t \lambda A}\right)=e^{i t f(\lambda A)}
$$

for every $t, \lambda \in \mathbb{R}$ which implies $\lambda f(A)=f(\lambda A)$. Consequently, $f: \mathscr{A}_{s} \rightarrow \mathscr{B}_{s}$ is a linear transformation. 
To obtain the last statement of the lemma, we use the fact that for any symmetry $V \in \mathscr{A}_{u}$ the element $\phi(V)$ is also a symmetry and compute

$$
\begin{gathered}
e^{i t \phi(V) f(A) \phi(V)}=\phi(V) e^{i t f(A)} \phi(V)=\phi(V) \phi\left(e^{i t A}\right) \phi(V) \\
=\phi\left(V e^{i t A} V\right)=\phi\left(e^{i t V A V}\right)=e^{i t f(V A V)} .
\end{gathered}
$$

Since this holds for every $t \in \mathbb{R}$ we deduce the desired equality $f(V A V)=$ $\phi(V) f(A) \phi(V)$ for every $A \in \mathscr{A}_{s}$ and symmetry $V \in \mathscr{A}_{u}$.

It remains to prove that $f$ preserves commutativity. Pick commuting elements $A, B \in \mathscr{A}_{s}$. Then for every $t, s \in \mathbb{R}$ we have

$$
e^{i t A} e^{i 2 s B} e^{i t A}=e^{i s B} e^{i 2 t A} e^{i s B}
$$

implying

and hence

$$
\phi\left(e^{i t A}\right) \phi\left(e^{i 2 s B}\right) \phi\left(e^{i t A}\right)=\phi\left(e^{i s B}\right) \phi\left(e^{i 2 t A}\right) \phi\left(e^{i s B}\right)
$$

$$
e^{i t f(A)} e^{i 2 s f(B)} e^{i t f(A)}=e^{i s f(B)} e^{i 2 t f(A)} e^{i s f(B)} .
$$

Fixing the real variable $s$ and putting the complex variable $z$ into the place of $i t$ we have that the equality

$$
e^{z f(A)} e^{i 2 s f(B)} e^{z f(A)}=e^{i s f(B)} e^{2 z f(A)} e^{i s f(B)}
$$

between von Neumann algebra valued holomorphic (entire) functions of the variable $z$ holds along the imaginary axis in the complex plane. By the uniqueness theorem of holomorphic functions we infer that the above equality necessarily holds on the whole plane. Next, fixing $z$ and inserting the complex variable $w$ into the place of $i s$, the same reasoning leads to that the equality

$$
e^{z f(A)} e^{2 w f(B)} e^{z f(A)}=e^{w f(B)} e^{2 z f(A)} e^{w f(B)}
$$

holds for all values of the variables $z, w \in \mathbb{C}$. In particular, for arbitrary real numbers $t$, s setting $z=t / 2, w=s / 2$ we have

$$
\sqrt{e^{t f(A)}} e^{s f(B)} \sqrt{e^{t f(A)}}=\sqrt{e^{s f(B)}} e^{t f(A)} \sqrt{e^{s f(B)}} .
$$

Just as in the last paragraph of the proof of Lemma 16 we infer from (23) that

$$
e^{t f(A)} e^{s f(B)}=e^{s f(B)} e^{t f(A)}
$$

holds for all $t, s \in \mathbb{R}$. This immediately implies $f(A) f(B)=f(B) f(A)$ which verifies that $f$ indeed preserves commutativity.

We are now in a position to prove Theorem 8 on the structure of continuous Jordan triple isomorphisms between unitary groups of von Neumann factors.

Proof of Theorem 8. Let $\phi: \mathscr{A}_{u} \rightarrow \mathscr{B}_{u}$ be a continuous Jordan triple isomorphism. By the first statement in Lemma 22 we know that $\phi(I)$ is a symmetry in $\mathscr{B}$ which commutes with $\mathscr{B}_{u}$ implying that it is a central element in $\mathscr{B}$. Considering the map $\psi()=.\phi(I)^{-1} \phi($.) we have a unital continuous Jordan triple isomorphism from $\mathscr{A}_{u}$ onto $\mathscr{B}_{u}$. Clearly this map and also its inverse send symmetries to symmetries. Apparently, for a symmetry $S$ and unitary $U$, we have $S, U$ commute if and only if $S U S=U$. This gives us that $\psi, \psi^{-1}$ preserve commutativity 
between an arbitrary symmetry and an arbitrary unitary. Hence both transformations send central unitaries to central unitaries (in fact, by spectral theorem it is apparent that a unitary is central if and only if it commutes with all symmetries). Since $\mathscr{A}$ is a factor, it has only two central symmetries. Therefore, the same must hold for $\mathscr{B}$, too. This means that $\mathscr{B}$ is also a factor and concerning the central symmetry $\phi(I)$ in $\mathscr{B}$ we have $\phi(I) \in\{I,-I\}$. Without loss of generality we may and do assume that $\phi(I)=I$ (in particular, we have $\psi=\phi$ ).

Before proceeding further let us consider the case where $\mathscr{A}$ if of type $\mathrm{I}_{1}$ (i.e., where $\mathscr{A}$ is isomorphic to $\mathbb{C}$ ). In that case all unitaries in $\mathscr{A}$ are central which implies that the same holds in $\mathscr{B}$, too. This means that $\mathscr{B}$ is commutative and hence it is also isomorphic to $\mathbb{C}$. The same argument applies when $\mathscr{B}$ is assumed to be of type $\mathrm{I}_{1}$ and yields that $\mathscr{A}$ must be of the same type, too. The structure of all continuous automorphisms of the circle group is well-known and one can trivially complete the proof in the particular case where one of $\mathscr{A}, \mathscr{B}$ is of type $\mathrm{I}_{1}$. So, in what follows we assume that $\mathscr{A}, \mathscr{B}$ are not of that type.

Next, by Lemma 22 we have a commutativity preserving linear transformation $f: \mathscr{A}_{s} \rightarrow \mathscr{B}_{s}$ such that

$$
\phi\left(e^{i t A}\right)=e^{i t f(A)}, \quad t \in \mathbb{R}, A \in \mathscr{A}_{s} .
$$

We claim that $f$ is bijective. Observe that this would be trivial if we knew that $\phi^{-1}$ is also a continuous Jordan triple isomorphism. Since continuity of the inverse has not been assumed, we have to find another way to show the bijectivity. By the injectivity of $\phi$ we easily obtain that $f$ is also injective. Since $\phi$ sends central unitaries to central unitaries, we obtain that $\exp (\operatorname{itf}(I))$ is a scalar (meaning that scalar times the identity) for every $t \in \mathbb{R}$ implying that $f(I)$ is also scalar which is nonzero by the injectivity of $f$. We show that every projection in $\mathscr{B}$ belongs to the range of $f$. To see this, pick an arbitrary projection $Q$ from $\mathscr{B}$. Then $\exp (i \pi Q)$ is a symmetry in $\mathscr{B}$ and since the symmetries in $\mathscr{A}_{u}$ are bijectively mapped onto the symmetries in $\mathscr{B}_{u}$, it follows that there is a projection $P$ in $\mathscr{A}$ such that

$$
e^{i \pi Q}=\phi\left(e^{i \pi P}\right)=e^{i \pi f(P)} .
$$

From this equality we infer that $f(P)=m I+n Q$ holds for some integers $m, n$. If $Q$ is a nontrivial projection, then $f(P)$ is obviously not a scalar. Therefore, we have $n \neq 0$ which implies that $Q$ is in the range of $f$. Therefore, all projections in $\mathscr{B}$ belong to that range. Since every element of a von Neumann factor is the finite linear combination of projections (e.g., see [10]), it follows that $f$ maps $\mathscr{A}_{s}$ onto $\mathscr{B}_{s}$. Consequently, $f$ is really bijective.

In the case where $\mathscr{A}$ is of type $\mathrm{I}_{n}$ ( $1<n$ is finite), referring to linear dimensions we obtain that $\mathscr{B}$ is of the same type and hence both algebras are isomorphic to $\mathbb{M}_{n}$. The statement of Theorem 8 for matrix algebras has been proven in [22], see Corollary 2. So in what follows we assume that none of $\mathscr{A}, \mathscr{B}$ is of type $\mathrm{I}_{n}, n$ being finite.

We continue as in the proof of Theorem 5. Namely, we extend $f$ from $\mathscr{A}_{s}$ onto $\mathscr{A}$ by the formula

$$
F(A+i B)=f(A)+i f(B) \quad\left(A, B \in \mathscr{A}_{s}\right)
$$


and obtain a normal preserving bijective linear map $F: \mathscr{A} \rightarrow \mathscr{B}$. Just as there we can infer that there is a nonzero real number $c$, an algebra *-isomorphism or an algebra *-antiisomorphism $\theta: \mathscr{A} \rightarrow \mathscr{B}$, and a linear functional $l: \mathscr{A} \rightarrow \mathbb{C}$ which is real valued on $\mathscr{A}_{S}$ such that

$$
F(A)=c \theta(A)+l(A) I \quad(A \in \mathscr{A}) .
$$

We have

$$
\phi\left(e^{i t A}\right)=e^{i t(c \theta(A)+l(A) I)}=e^{i t l(A)} \theta\left(e^{i t c A}\right), \quad t \in \mathbb{R}, A \in \mathscr{A}_{S} .
$$

It follows that $\phi(\exp (i t A))$ is a scalar multiple of $\theta(\exp (i t c A))$ for every $t \in \mathbb{R}$ and $A \in \mathscr{A}_{s}$. We claim that $c= \pm 1$. To verify this, we again recall that $\phi$ sends symmetries to symmetries. Let $P$ be a nontrivial projection in $\mathscr{A}$. Then $\exp (i \pi P)$ is a symmetry and it follows that the symmetry $\phi(\exp (i \pi P))$ is a scalar multiple of $\theta(\exp (i \pi c P))=\exp (i \pi c \theta(P))$, where $\theta(P)$ is a nontrivial projection. It is easy to see that the scalar multiplier in question is necessarily \pm 1 and then we obtain that the number $\exp (i \pi c)$ also equals \pm 1 . From this we get that $c$ is an integer, say $c=m$. Since every element of $\mathscr{A}_{u}$ is of the form $\exp (i A)$ with some $A \in \mathscr{A}_{s}$, we thus obtain from (25) that $\phi(V)$ is a scalar multiple of $\theta\left(V^{m}\right)$ for every $V \in \mathscr{A}_{u}$.

By the Jordan triple multiplicativity of $\phi$ and $\theta$ this gives us that the nonzero integer $m$ has the property that $(V W V)^{m}$ and $V^{m} W^{m} V^{m}$ are scalar multiples of each other whenever $V, W \in \mathscr{A}_{u}$. Applying Proposition 18 we infer that $m= \pm 1$.

Using (25) we can write

$$
\phi(U)=\varphi(U) \theta\left(U^{m}\right), \quad U \in \mathscr{A}_{u},
$$

where the functional $\varphi: \mathscr{A}_{u} \rightarrow \mathbb{T}$ satisfies

$$
\varphi\left(e^{i t A}\right)=e^{i t l(A)}, \quad t \in \mathbb{R}, A \in \mathscr{A}_{s} .
$$

Clearly, $\varphi$ is a continuous Jordan triple map with values in the circle group and we have $\varphi(I)=1$. It follows that $\varphi(S)= \pm 1$ for any symmetry $S \in \mathscr{A}_{u}$. Applying the last assertion in Lemma 22 in the particular case where $\mathscr{B}=\mathbb{C}$, we obtain that $l(S A S)=l(A)$ holds for every $A \in \mathscr{A}_{s}$ and symmetry $S \in \mathscr{A}_{u}$. By linearity the same holds for any $A \in \mathscr{A}$, too. Then just as in the proof of Lemma 17 we deduce for any $X \in \mathscr{A}$ and symmetry $S \in \mathscr{A}_{u}$ that $l(S X)=l(S(X S) S)=l(X S)$ implying $l(P X)=l(X P)$ whenever $P \in \mathscr{A}$ is a projection and $X \in \mathscr{A}$. Using the fact that any factor as a linear space is generated by its projections, it follows that $l$ is a tracial linear functional on $\mathscr{A}$. We have mentioned above that $\varphi(S)= \pm 1$ holds for any symmetry $S \in \mathscr{A}_{u}$. It follows that

$$
\pm 1=\varphi\left(e^{i \pi P}\right)=e^{i \pi l(P)}
$$

which implies that the value $l(P)$ is an integer for every projection $P \in \mathscr{A}$. Since $l$ is a tracial linear functional on $\mathscr{A}$, it takes equal values on equivalent projections. If $\mathscr{A}$ is of one of the types $\mathrm{II}_{1}, \mathrm{II}_{\infty}, \mathrm{III}$, then any nonzero projection $P$ in $\mathscr{A}$ can be written as the sum of an arbitrary finite number of equivalent projections. It follows that the integer $l(P)$ is divisible by any positive integer and this implies that $l(P)=0$. If $\mathscr{A}$ is of type $\mathrm{I}_{\infty}$, in the same way we obtain that $l(P)=0$ holds for any infinite projection and then refer to the fact that any finite projection is the 
difference of two infinite ones. In all those cases we can infer that $l$ vanishes on the set of all projections in $\mathscr{A}$ which then implies that $l$ is zero everywhere.

Therefore, we have $\phi(U)=\theta\left(U^{m}\right), U \in \mathscr{A}_{u}$ where $m= \pm 1$ and this completes the proof.

We now can easily prove our last result Theorem 9.

Proof of Theorem 9. First observe that $\phi$ is also injective. We next define $\psi(U)=$ $\phi(I)^{-1} \phi(U), U \in \mathscr{A}_{u}$. It is apparent that this bijective map also satisfies (8). By Theorem $7 \psi$ is a continuous inverted Jordan triple isomorphism. But it is a unital map and hence easily follows that it is necessarily a continuous Jordan triple isomorphism. Applying Theorem 8 one can complete the proof readily.

Remark 23. We conclude with a few remarks.

Notice that in our results on the structure of continuous Jordan triple isomorphisms as well as on that of the "generalized isometries" between positive definite cones we have assumed that the underlying algebras are not of type $\mathrm{I}_{2}$ (while there has not been such an assumption relating to unitary groups). The reason is connected to the use of the structural result concerning normal preserving maps which does not hold in algebras of type $\mathrm{I}_{2}$. In the case of unitary groups, referring to a result in [22] we could handle that situation but, unfortunately, we have not been able to do so in the case of the positive definite cone. So, it might be rather surprising, but we do not have a proof in the very particular case represented by $2 \times 2$ matrices. In fact, we believe the problem is far not so simple as one might think at the first sight. We leave this as an open problem.

Finally, we emphasize that our results on "generalized isometries" are not "if and only if" type results. Indeed, they assert that every invariance transformation under consideration is of a certain form but it is not necessary that all maps of those given forms have the corresponding invariance properties. This is due to the generality of the circumstances in those results. Hence, in concrete situations one needs to go further and select from the groups of transformations that appear in the conclusions of our results those ones which really have the actual invariance property.

\section{REFERENCES}

1. W.N. Anderson and G.E. Trapp, Operator means and electrical networks, Proc. 1980 IEEE International Symposium on Circuits and Systems (1980), 523-527.

2. R. Beneduci and L. Molnár, On the standard K-loop structure of positive invertible elements in $a C^{*}$-algebra, preprint.

3. R. Bhatia, Matrix Analysis, Graduate Texts in Mathematics 169, New York, Springer, (1996).

4. A.M. Bikchentaev, On a property of $L_{p}$-spaces on semifinite von Neumann algebras, Math. Notes 64 (1998), 159-163.

5. F. Botelho, J. Jamison and L. Molnár, Surjective isometries on Grassmann spaces, J. Funct. Anal. 265 (2013), 2226-2238.

6. K.I. Beidar, M. Brešar, M.A. Chebotar and Y. Fong, Applying functional identities to some linear preserver problems, Pacific J. Math. 204 (2002), 257-271. 
7. J.T. Chan, C.K. Li and C.C.N. Tu, A class of unitarily invariant norms on B(H), Proc. Amer. Math. Soc. 129 (2001), 1065-1076.

8. H.F. Chau, C.K. Li, Y.T Poon and N.S. Sze, Induced metric and matrix inequalities on unitary matrices, J. Phys. A: Math. Theor. 45 (2012), 095201, 8 pp.

9. B. Fuglede and R.V. Kadison, Determinant theory in finite factors, Ann. of Math. 55 (1952), 520-530.

10. S. Goldstein and A. Paszkiewicz, Linear combinations of projections in von Neumann algebras, Proc. Amer. Math. Soc. 116 (1992), 175-183.

11. H. Halpern, Commutators in properly infinite von Neumann algebras, Trans. Amer. Math. Soc. 139 (1969), 55-73.

12. O. Hatori, G. Hirasawa, T. Miura and L. Molnár, Isometries and maps compatible with inverted Jordan triple products on groups, Tokyo J. Math. 35 (2012), 385-410.

13. O. Hatori and L. Molnár, Isometries of the unitary group, Proc. Amer. Math. Soc. 140 (2012), 2141-2154.

14. O. Hatori and L. Molnár, Isometries of the unitary groups and Thompson isometries of the spaces of invertible positive elements in $C^{*}$-algebras, J. Math. Anal. Appl. 409 (2014), 158-167.

15. S. Honma and T. Nogawa, Isometries of the geodesic distances for the space of invertible positive operators and matrices, Linear Algebra Appl. 444 (2014), 152-164.

16. H. Hotje, M. Marchi and S. Pianta, On a class of point-reflection geometries, Discrete Math. 129 (1994), 139-147.

17. R.V. Kadison and J.R. Ringrose, Fundamentals of the Theory of Operator Algebras, Vol II., Academic Press, 1986.

18. H. Karzel, M. Marchi and S. Pianta, On commutativity in point-reflection geometries, J. Geom. 44 (1992), 102-106.

19. C.F Manara and M. Marchi, On a class of reflection geometries, Istit. Lombardo Accad. Sci. Lett. Rend. A 125 (1991), 203-217.

20. L. Molnár, A remark to the Kochen-Specker theorem and some characterizations of the determinant on sets of Hermitian matrices, Proc. Amer. Math. Soc. 134 (2006) 2839-2848.

21. L. Molnár, Thompson isometries of the space of invertible positive operators, Proc. Amer. Math. Soc. 137 (2009), 3849-3859

22. L. Molnár, Jordan triple endomorphisms and isometries of unitary groups, Linear Algebra Appl. 439 (2013), 3518-3531.

23. L. Molnár, Jordan triple endomorpisms and isometries of spaces of positive definite matrices, Linear and Multilinear Alg., to appear. DOI:10.1080/03081087.2013.844231

24. L. Molnár, A few conditions for a $C^{*}$-algebra to be commutative, preprint.

25. L. Molnár and P. Šemrl, Transformations of the unitary group on a Hilbert space, J. Math. Anal. Appl. 388 (2012), 1205-1217.

26. L. Molnár and P. Szokol, Transformations on positive definite matrices preserving generalized distance measures, submitted.

MTA-DE "LendÜlet" Functional Analysis Research Group, Institute of MathematICS, University of Debrecen, H-4010 Debrecen, P.O. BoX 12, Hungary

E-mail address: molnarl@science. unideb.hu

URL: http://www. math. unideb.hu/ molnarl/ 